\title{
Contribution to the knowledge of Hymenoptera from the Aeolian Archipelago (Sicily), emphasizing Aculeata
}

\author{
Giuseppe Fabrizio Turrisi ${ }^{*}$, Giovanni Altadonna ${ }^{2}$, Pietro Lo Cascio ${ }^{3}$, Vittorio Nobile ${ }^{4}$ Marco Selis ${ }^{5}$ \\ ${ }^{1}$ Via Cristoforo Colombo 8, 95030 Pedara, Italy; e-mail: giuseppefabrizioturrisi@gmail.com \\ ${ }^{2}$ Contrada Filangeri s.n.c., Vill. Pistunina, 98125 Messina, Italy; e-mail: altadonnagiovanni415@gmail.com \\ ${ }^{3}$ Nesos, via Vittorio Emanuele 24, 98055 Lipari, Messina, Italy; e-mail: plocascio.nesos@gmail.com \\ ${ }^{4}$ Via Psaumida, 17, lotto 25, 97100 Ragusa, Italy; e-mail: nobilevittorio40@gmail.com \\ ${ }^{5}$ Via dei Tarquini 22, 01100 Viterbo, Italy; e-mail: marcozetsu@hotmail.it \\ *Corresponding author
}

\begin{abstract}
This study provides the first contribution to the knowledge of the Order Hymenoptera, emphasizing Aculeata, for the Aeolian archipelago, based on recent field investigations and examination of several collections. A total of 169 species belonging to 19 families are recorded: Evaniidae (1 species), Aulacidae (2 species), Gasteruptiidae (3 species), Ichneumonidae (1 species), Leucospidae (1 species), Chrysididae ( 8 species), Tiphiidae ( 3 species), Scoliidae (6 species), Mutillidae (13 species), Pompilidae (6 species), Vespidae (14 species), Sphecidae (6 species), Crabronidae (24 species), Formicidae (14 species), Colletidae (4 species), Andrenidae (8 species), Halictidae (29 species), Megachilidae (12 species), Apidae (14 species). Most part of species are newly recorded for the Aeolian Archipelago, the following being new records for Sicily: Dryudella esterinae Pagliano, 2001, Nysson quadriguttatus Spinola, 1808, Miscophus aetoni Saunders, 1903, Miscophus lusitanicus Andrade, 1952, Cerceris circularis dacica Schletterer, 1887 (Crabronidae), and Lasioglossum (Dialictus) semilucens (Alfken, 1914) (Halictidae); Miscophus lusitanicus is also newly recorded for Italy. Combining the available data, a total of 218 species of Hymenoptera are currently known for the Aeolian Archipelago. The Hymenoptera fauna of these islands is dominated by widespread Mediterranean or West Palaearctic species, and most of them are also found in the Western Mediterranean Basin. The study briefly highlights some key points regarding biogeographical and conservation of this group and its significant role in the management of ecosystems in the Aeolian Archipelago.
\end{abstract}

KEY WORDS Aeolian Archipelago; Sicily; Hymenoptera; faunal list.

Received 21.05.2020; accepted 02.07.2020; published online 30.09.2020

\section{INTRODUCTION}

The Aeolian Islands have great importance in terms of environmental and biological heritage, and have been recognized by UNESCO as World Heritage Site. This archipelago harbors five protected areas and several sites of the Nature 2000 network, and has been also proposed as National Park in 2007 , although that has not yet been followed by a real institution. Both fauna and flora of these islands are characterized by the occurrence of many species of biogeographical and conservation interest, including some endemics. The faunal knowledge may be considered exhaustive for vertebrates, while several 
groups of invertebrates remain still less investigated (Lo Cascio, 2017).

Among them, the hymenopterans have been only poorly studied despite their ecological importance, e.g., for the pollination of wild plants and crops as well as for the biological control of many groups of Arthropods. Data on the Hymenoptera from these islands are scanty and scattered in a wide literature, which includes monographs, collection's and taxonomic reviews (Santschi, 1927, 1934; Baroni Urbani, 1964, 1971; Warncke, 1981; Pagliano, 1987; Gayubo et al., 1988; Matteini Palmerini, 1992; Pagliano \& Nobile, 1993; Ebmer, 1995; Olmi, 1999; Sanetra et al., 1999; Czösz \& Schulz, 2010; Vicidomini et al., 2005; Boni Bartalucci, 2012; Pavesi \& Rosa, 2013; Borowiec et al., 2015; Turrisi et al., 2015; Seifert, 2016; Korenko, 2017; Scupola, 2019), the "Checklist of Italian fauna" (Comba \& Comba, 2005; Generani et al., 2005; Olmi, 2005; Strumia, 2005), papers related to apicolture and/or biological control (Monastero \& Genduso, 1964a, 1964b; Monastero, 1965; Monastero \& Delaonoue, 1965, 1966a, 1966b, Genduso \& Mineo, 1974; Dall’Olio et al., 2008; Daane \& Johnson, 2010; Bouga et al., 2011; Tenore et al., 2012; Muñoz et al., 2014; Utzeri et al., 2018) and faunal papers concerning Italian peninsular and insular areas or other islands (Kutter, 1927; Baccetti, 1967; Focarile, 1972; Nobile \& Turrisi, 1996; Lo Cascio et al., 1998; Mei, 1998, 2008; Turrisi, 1999; Lo Cascio \& Navarra, 2003; Borsato \& Turrisi, 2004; Lo Cascio, 2004, 2015; Lo Cascio \& Romano, 2004; Lo Cascio et al., 2006; Jucker et al., 2008; Ceccolini \& Barbagli, 2014; Queiroz \& Alves, 2016; Turrisi \& Altadonna, 2017; Korenko \& Di Giovanni, 2019), while just few papers are specifically referred to the fauna of the Aeolian Archipelago, although not exclusively dedicated to Hymenoptera (Costantino, 1937; Lo Cascio, 2004).

The aim of this paper is to update the knowledge of the Hymenoptera of the Aeolian Archipelago through the study of the material from several private and museum collections and that collected during recent field surveys.

\section{MATERIAL AND METHODS}

\section{Study area}

The Aeolian Archipelago (Fig. 1) lies between $38^{\circ} 21^{\prime} 54^{\prime \prime}$ and $38^{\circ} 48^{\prime} 40^{\prime \prime}$ latitude North and $14^{\circ} 20^{\prime} 35^{\prime \prime}$ and $15^{\circ} 14^{\prime} 70^{\prime \prime}$ longitude East of Greenwich, in the southern Tyrrhenian Sea. It includes seven main islands (Lipari, Salina, Vulcano, Stromboli, Filicudi, Alicudi and Panarea) whose surface ranges from 37.29 to $3.34 \mathrm{~km} 2$, and several uninhabited islets and rocks. The sourthernmost island, Vulcano, is located $20 \mathrm{~km}$ from the Sicily, while the northernmost, Stromboli, is only 55 far from the Italian Peninsula (Capo Vaticano). The origin of this volcanic archipelago is strictly linked to the subduction processes related to the convergence between African and Euro-Asiatic plates. The islands are disposed along a semi-arc and represents the top of a wide volcanic system, that includes also several sea-mounts. The beginning of the activity in this area is dated back about 1,000,000 years ago, but the actual islands emerged during the last 300,000 years and some of them (for instance, Alicudi, Stromboli and Vulcano) are rather "young", with an estimated age of less than 100,000 years. Currently there are only three active volcanoes, namely Stromboli, Gran Cratere or La Fossa on Vulcano and a sub-marine crater off the eastern coast of Panarea. The first human settlements in the archipelago are dated to the end of 6th millennium B.C., hence the islands have undergone to a longterm anthropization; this trait play a significant role in their actual landscape, that is widely characterized by terraced agricultural lands, mostly abandoned since the dramatic economical crisis that involved the local communities between late 19th and the first half of the 20th century. Currently these areas host a complex mosaic of xeric grasslands, garrigues and maquis, with more mature and structurated spots of the latter occurring on the top and the inner part of some islands (Salina, Lipari, Filicudi) (Lo Cascio, 2017).

\section{Materials}

Data provided in the present paper include the records given in literature and those obtained from the museum and private collections of the authors and several colleagues. The most part of the field surveys was done on the islands of Lipari and Vulcano, but some investigations were carried out on other islands. The specimens were gathered mainly through traditional collecting-methods (hand net), but also yellow-pan traps, Moericke traps, wine 


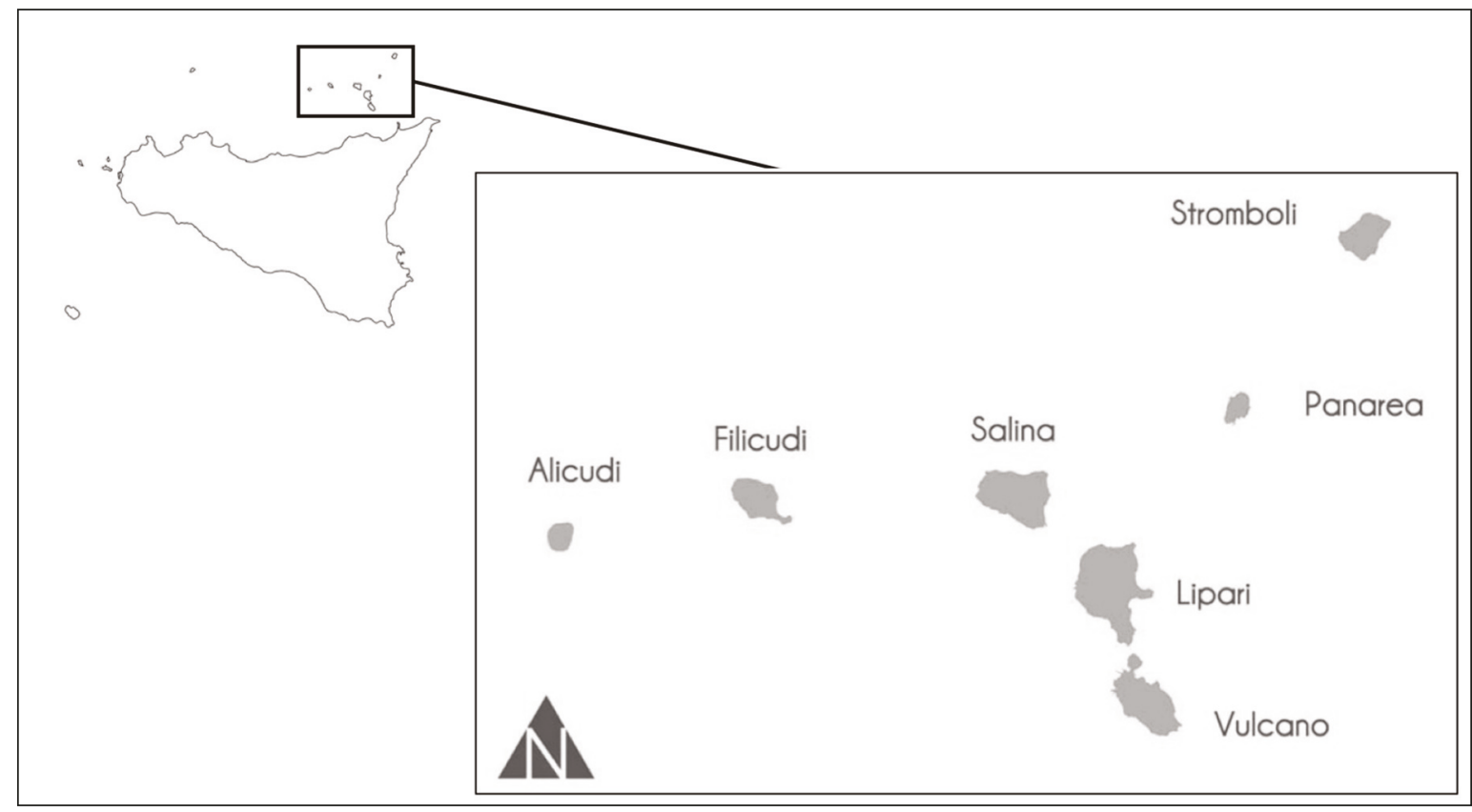

Figure 1. Localization of the study area.

traps and light traps have been used. For each species treated, we provide:

1) list of material examined;

2) distribution;

3) remarks, including eventual previous records.

The list of species is arranged according to the "Checklist della Fauna d'Italia" (Ruffo \& Stock, 2005).

ACRONYMS. GAC: Giovanni Altadonna, Messina, Italy; WBC: Walter Borsato, Verona, Italy; DSCC: Dipartimento Scienze Biologiche, Geologiche e Ambientali, Università di Catania, Sezione Biologia Animale, Italy; PLCC: Pietro Lo Cascio, Lipari, Italy; MMC: Maurizio Mei, Roma, Italy; RAC: Museo Regionale di Terrasini (Coll. R. Alliata), Terrasini (Palermo), Italy; PNC: Pietro Niolu, Sassari, Italy; VNC: Vittorio Nobile, Ragusa, Italy; MRC: Marcello Romano, Capaci (Palermo), Italy; MSC: Marco Selis, Viterbo, Italy; GFTC: Giuseppe FabrizioTurrisi, Pedara (Catania), Italy; CVC: Carlo Violani, Milan, Italy.

\section{RESULTS}

A total of 169 species are herein recorded for the Aeolian Archipelago, belonging to 19 families:
Evaniidae (1 species), Aulacidae (2 species), Gasteruptiidae (3 species), Ichneumonidae (1 species), Leucospidae (1 species), Chrysididae (8 species), Tiphiidae (3 species), Scoliidae (6 species), Mutillidae (13 species), Pompilidae (6 species), Sphecidae (6 species), Crabronidae (24 species), Vespidae (14 species), Formicidae (14 species), Colletidae (4 species), Andrenidae (8 species), Halictidae (29 species), Megachilidae (12 species), Apidae (14 species). A summary of the distribution of Hymenoptera on the Aeolian Archipelago, including records from literature, is provided in Table 1.

\section{Faunal list}

\section{EVANIIDAE}

Zeuxevania splendidula (Costa, 1884) (Fig. 2)

EXAMINED MATERIAL. Lipari: Pianoconte, 7.VI. 1997, 3 females, G.F. Turrisi leg. and det. (GFTC).

Distribution. South European.

REMARKS. New for the Aeolian Archipelago.

\section{AULACIDAE}


Pristaulacus galitae (Gribodo, 1879)

EXAMINED MATERIAL. Vulcano: 28.VI.18. VII. 2008, 2 males, 2 females, M. Mei leg., G.F. Turrisi det. (GFTC).

Distribution. West Palaearctic.

Remarks. New for the Aeolian Archipelago, known from many other Mediterranean islands, e.g., Maltese Islands (Turrisi, 2007; Turrisi \& Rattu, 2019).

Pristaulacus lindae Turrisi, 2000 (Fig. 3)

EXAMINED MATERIAL. Vulcano: 28.VI.-18.VII. 2008, 1 female, M. Mei leg., G.F. Turrisi det. (GFTC).

Distribution. South West European.

Remarks. New for the Aeolian Archipelago. Described from Sicily (Turrisi, 2000), subsequently recorded from other localities from Eastern Sicily (Turrisi, 2007), it has recently been recorded from Iberian Peninsula (Carbonell-Font \& Turrisi, 2017).

\section{GASTERUPTIIDAE}

Gasteruption assectator (Linnaeus, 1758)

EXAMINED MATERIAL. Lipari: Castellaro, 23. VI. 1998, 1 female, B. Carletti, B. Cecchi, L. Dapporto, P. Lo Cascio, C. Moreno leg., G.F. Turrisi det. (PLCC).

Distribution. Holoarctic.

Remarks. New for the Aeolian Archipelago.

Gasteruption erythrostomum (Dahlbom, 1831)

EXAMIned MATERIAL. Lipari: Urnazzo, 2. VI. 1997, 1 female, P. Lo Cascio leg., G.F. Turrisi det. (PLCC).

DisTRIBUTION. European.

REMARKS. New for the Aeolian Archipelago.

Gasteruption jaculator (Linnaeus, 1758)

EXAMINED MATERIAL. Lipari: Pianoconte, 7.
VII. 1999, 1 female, G.F. Turrisi leg. and det. (GFTC).

Distribution. West Palaearctic.

REMARKS. New for the Aeolian Archipelago.

\section{ICHNEUMONIDAE}

Eutanyacra picta (Schrank, 1776) (Fig. 4)

EXAMINED MATERIAL. Vulcano: Piano, 4. VI. 1997, 1 female, P. Lo Cascio leg., G.F. Turrisi det. (PLCC).

Distribution. Palaearctic and Oriental.

REMARKS. New for the Aeolian Archipelago.

\section{LEUCOSPIDAE}

Leucospis gigas Fabricius, 1793 (Fig. 5)

EXAMINED MATERIAL. Lipari: Pianoconte, 7. VI. 1997, G.F. Turrisi leg. and det. (GFTC).

DisTRIBUTION. European Asian (southern part). The record from Nearctic requires confirmation.

REMARKS. New for Lipari, previously observed and photographed in Stromboli on 12.06.2011 (https://www.naturamediterraneo.com/forum/topic. asp?TOPIC_ID=146201). It is primarily associated with Megachilidae (Noyes, 2019), and among the known hosts there are two large Megachile (Chalicodoma): M. (C.) parietina (Geoffroy, 1785) and M. (C.) sicula Rossi, 1792, both collected in Lipari, the former one in the same place and in the same time along a dry-stone wall.

\section{CHRYSIDIDAE}

Hedychridium cf. mediocrum Linsenmaier, 1987

EXAMINED MATERIAL. Salina: Monte Fossa delle Felci, VII.2017, P. Lo Cascio leg., P. Rosa det. (PLCC).

Distribution. South European.

REMARKS. New for the Aeolian Archipelago and Sicily. 
Hedychrum cf. micans ssp. europaeum Linsenmaier, 1959

EXAMined MATERIAL. Salina: Monte Fossa delle Felci, 22.V.2009, P. Lo Cascio \& F. Grita leg., P. Rosa det. (PLCC).

Distribution. West European.

Remarks. New for the Aeolian Archipelago.

Holopyga fervida (Fabricius, 1781) (Fig. 6)

EXAMINED MATERIAL. Alicudi: VII.2013, 2 females, P. Lo Cascio \& F. Grita leg., P. Rosa det. (PLCC). Vulcano, crater, VII.2016, 1 female, P. Lo Cascio leg., P. Rosa det. (PLCC); Lipari, Palmeto, V.2016, 1 female, P. Lo Cascio leg., P. Rosa det. (PLCC). Panarea, Soldata, 20.V.2009, 1 female, P. Lo Cascio \& F. Grita leg., P. Rosa det. (PLCC). Stromboli, Nel Cannestra, V.2016, 1 female, P. Lo Cascio leg., P. Rosa det. (PLCC).

Distribution. West Palaearctic.

Remarks. New for the Aeolian Archipelago.

Pseudomalus auratus (Linnaeus, 1758)

EXAMINED MATERIAL. Lipari: Castello environs, 8.VI.1997, 1 male, G.F. Turrisi leg. and det. (GFTC). Lipari: Palmeto, 20.VI.1998, 1 female, B. Carletti et al. leg., G.F. Turrisi det. (PLCC). Lipari: Monte Guardia, IV.2009, 3 females, P. Lo Cascio \& F. Grita leg., reared from wood, G.F. Turrisi det. (PLCC).

Distribution. Holarctic.

REMARKS. Recently recorded from Lipari by Pavesi \& Rosa (2013).

Chrysis cf. auriceps Mader, 1936

EXAmined Material. Panarea: Soldata, $230 \mathrm{~m}$ a.s.1., 21.VIII.2015, 2 specs., G. Altadonna leg., yellow-pan trap, P. Rosa det. (GAC).

DisTRIBUTION. Mediterranean.

Remarks. New for the Aeolian Archipelago.

Chrysis cerastes Abeille De Perrin, 1877 (Fig. 7)

EXAMINED MATERIAL. Salina: Monte Fossa delle
Felci, VII.2017, 1 female, P. Lo Cascio leg., P. Rosa det. (PLCC).

Distribution. South European.

REMARKs. New for the Aeolian Archipelago.

Chrysis scutellaris Fabricius, 1794

EXAmined Material. Filicudi: Capo Graziano, 26.VI.2016, 1 male, P. Lo Cascio leg., P. Rosa det. (PLCC).

Distribution. West European.

REMARKS. New for the Aeolian Archipelago.

Pseudochrysis neglecta (Shuckard, 1837)

EXAMINED MATERIAL. Vulcano: Piano, 20. IV. 2009, 1 spec., P. Lo Cascio \& F. Grita leg., P. Rosa det. (PLCC).

Distribution. European Asian

REMARKS. New for the Aeolian Archipelago.

\section{TIPHIIDAE}

Tiphia lepeletieri Berland, 1925

EXAMINED MATERIAL. Lipari: Monte Chirica, 26. IX.1996, 1 female, P. Lo Cascio leg., G.F. Turrisi det. (PLCC); Vallone Muria, VII.1999, 1 male, Moericke trap, P. Lo Cascio leg., G.F. Turrisi det. (PLCC); Castellaro, IX.1998, 1 male, P. Lo Cascio leg., G.F. Turrisi det. (PLCC).

DisTRIBUTION. Italian.

REMARKS. Known from Sicily (Boni Bartalucci, 2012, 2013), new for the Aeolian Archipelago.

Meria dorsalis (Fabricius, 1804) (Fig. 8)

EXAMINED MATERIAL. Lipari: Urnazzo, 30. VII. 1996, 1 female, P. Lo Cascio leg., G.F. Turrisi det. (PLCC); Chiesa Vecchia, 7.VII.1999, 2 females, on flowers of cultivated Petroselinum, G.F. Turrisi leg. and det. (GFTC). Vulcano: Piano, $330 \mathrm{~m}$ a.s.l., 18.VIII.2015, 1 female, G. Altadonna leg., G.F. Turrisi det. (GAC); 9-22.VIII.2015, 2 females, G. Altadonna leg., yellow-pan trap, G.F. Turrisi det. (GAC). 
Distribution. European Asian.

REMARKS. Recorded from Lipari by Boni Bartalucci (2012), is newly recorded for Vulcano.

Meria tripunctata (Rossi, 1790)

EXAMINED MATERIAL. Lipari: Vallone Muria, VII.1999, 1 female, P. Lo Cascio leg., Moericke trap, G.F. Turrisi det. (PLCC). Vulcano: Piano, 330 m a.s.1., 13.VIII.2015, 1 female, G. Altadonna leg., G.F. Turrisi det. (GAC); 9-22.VIII.2015, 3 females, G. Altadonna leg., yellow-pan trap, M. Selis det. (GAC).

Distribution. West Mediterranean.

REMARKS. Recorded from Lipari by Boni Bartalucci (2012), is newly recorded for Vulcano.

\section{SCOLIIDAE}

Megascolia (Regiscolia) maculata ssp. flavifrons (Fabricius, 1775)

EXAmined Material. Lipari: Pianoconte, 7. VI. 1997, 1 male, G.F. Turrisi leg. and det. (GFTC); Vulcano: Piani di Luccia, 2.VI.2012, 1 male, G. Altadonna leg. and det. (GAC).

Distribution. South European.

REMARKS. Recorded for Lipari and Salina by Generani et al., (2005) and for Vulcano, as Megascolia maculata (Drury, 1773), by Ceccolini \& Barbagli (2014).

Megascolia bidens (Fabricius, 1775)

Examined Material. Vulcano: Piano, 2. VI. 2011, 2 males, G. Altadonna leg. and det. (GAC)

Distribution. Mediterranean.

REMARKS. Recorded for Lipari and Vulcano by Generani et al. (2005).

Scolia hirta (Schrank, 1781)

EXAMINED MATERIAL. Lipari: Castello environs, 8.VI.1997, 1 male, G.F. Turrisi leg. and det. (GFTC).
Distribution. South West Palaearctic.

REMARKS. New for the Aeolian Archipelago.

Scolia carbonaria (Linnaeus, 1767) (Fig. 9)

EXAmined Material. Lipari: Pianoconte, 7. VI.1997, 1 male, G.F. Turrisi leg. and det. (GFTC). Lipari: Castello environs, 8.VI.1997, 1 male, G.F. Turrisi leg. and det. (GFTC).

Distribution. South Italian Maghrebian.

REMARKS. New for the Aeolian Archipelago.

Colpa (Colpa) sexmaculata (Fabricius, 1781)

EXAMINED MATERIAL. Vulcano: Monte Aria, 405 m a.s.1., 16.VIII.2014, 2 males, G. Altadonna leg., G.F. Turrisi det. (GAC, MSC).

Distribution. Southern Europe.

REMARKS. Previously recorded for "Stromboli, loc. Malpasseddu (Lipari, ME) 13.VII.2000, F. Barbagli \& S. Lotti [leg.]" and "Lipari, Monte Guardia (ME) 25.VIII.1996, P. Lo Cascio [leg.]” by Ceccolini \& Barbagli (2014), is newly recorded for Vulcano.

\section{Dasyscolia ciliata (Fabricius, 1787)}

ExAmined MATERial. Vulcano: Piano, $330 \mathrm{~m}$ a.s.1., 10.VIII.2013, 1 male, G. Altadonna leg., G.F. Turrisi det. (GAC).

Distribution. Mediterranean.

REMARKs. New for the Aeolian Archipelago.

\section{MUTILLIDAE}

Bidecoloratilla leopoldina (Invrea, 1955)

EXAMined MATERIAL. Lipari: Quattropani, 7. VI.1997, 1 male, 1 female, G.F. Turrisi leg. and det. (GFTC) (quoted in Turrisi, 1999, and Turrisi et al., 2015); Vallone Muria, IX.1995, VII-VIII.1996, 3.VI.1997, 3.VIII.1997, 9 males, 12 females, P. Lo Cascio leg., P. Lo Cascio \& M. Romano det. (PLCC); Madoro, 21.VIII.1996, 25.II.1996, 8 males, 5 females, P. Lo Cascio \& S. Pasta leg., P. Lo Cascio \& M. Romano det. (PLCC); Falcone, 
30.III.1997, 1 female, P. Lo Cascio leg., P. Lo Cascio \& M. Romano det. (PLCC); Timpone Ricotta, 3.VI.1997, 1 male, 6 females, P. Lo Cascio leg., P. Lo Cascio \& M. Romano det. (PLCC) (quoted in Lo Cascio \& Romano, 2004).

Distribution. West Mediterranean.

REMARKS. Recorded as Blakeius leopoldinus (Invrea, 1955) for Lipari by Turrisi (1999) and Lo Cascio \& Romano (2004); for Salina and Vulcano by Lo Cascio (2015).

\section{Myrmilla (Myrmilla) calva (Villers, 1789)}

EXAMINED MATERIAL. Lipari: Cappero, 19.VII.1995, 2 females, P. Lo Cascio \& S. Pasta leg., P. Lo Cascio \& M. Romano det. (PLCC); Timpone Ospedale, 18.IV.1996, 1 female, P. Lo Cascio leg., P. Lo Cascio \& M. Romano det. (PLCC); Vallone Muria, VII.-VIII.1996, 3.VI.1997, 8 females, P. Lo Cascio leg., P. Lo Cascio \& M. Romano det. (PLCC); Madoro, 21.VIII.1996, 3 females, P. Lo Cascio \& S. Pasta leg., P. Lo Cascio \& M. Romano det. (PLCC); Timpone Ricotta, VIII.1996, 3.VI.1997, 4.X.1997, 27.V.1998, 20.VI.1998, 1 male, 13 females, P. Lo Cascio leg. et al., P. Lo Cascio \& M. Romano det. (PLCC). Vulcano: Capo Grillo, 27.V.1983, 1 female, M. Romano leg., P. Lo Cascio \& M. Romano det. (MRC); Gran Cratere, 4.VI. 1997, 2 females, P. Lo Cascio leg., P. Lo Cascio \& M. Romano det. (PLCC) (this material is quoted in Lo Cascio \& Romano, 2004).

DisTRIBUTION. European Mediterranean.

REMARKS. Recorded for Lipari and Vulcano by Lo Cascio \& Romano (2004), quoted in Lo Cascio (2015).

Ronisia brutia ssp. brutia (Petagna, 1787) (Fig. 10)

EXAmined MATERIAL. Lipari: Monte Sant'Angelo, 28.VI.1966, 1 female, V. Nobile leg., G.F. Turrisi det. (quoted in Turrisi, 1999); Cappero, 25.V.1996, 1 female, P. Lo Cascio leg., P. Lo Cascio \& M. Romano det. (PLCC); Palmeto, 10.VII.1996, 1 female, A. Nistri \& S. Vanni leg., P. Lo Cascio \& M. Romano det. (CVC); Quattropani, 3.V.2001, 1 female, P. Lo Cascio leg., P. Lo Cascio \& M. Romano det. (PLCC). Filicudi, Zucco Grande,
6.V.2002, P. Lo Cascio leg., P. Lo Cascio \& M. Romano det. (PLCC) (this material is quoted in Lo Cascio \& Romano, 2004).

Distribution. South European.

REMARKS. Recorded for Lipari by Matteini Palmerini (1992) and Turrisi (1999), Lipari and Filicudi by Lo Cascio \& Romano (2004), Lipari, Vulcano, Salina, Filicudi and Panarea by Lo Cascio (2015).

Ronisia ghilianii (Spinola, 1843)

EXAMINED MATERIAL. Lipari: 3.X.1976, 1 female, B. Massa leg., P. Lo Cascio \& M. Romano det. (MRC); Cappero, VII.1995, 1 female, P. Lo Cascio leg., P. Lo Cascio \& M. Romano det. (PLCC); Vallone Muria, VII.1995, 13.VIII.1996, 3.VI.1997, 4 females, P. Lo Cascio leg., P. Lo Cascio \& M. Romano det. (PLCC); Timpone Ricotta, 3.VI.1997, 1 female, P. Lo Cascio leg., P. Lo Cascio \& M. Romano det. (PLCC); Palmeto, VIII.1998, 1 female, P. Lo Cascio leg., P. Lo Cascio \& M. Romano det. (PLCC). Panarea: Punta del Corvo, 26.V.2000, 1 female, P. Lo Cascio \& C. Moreno Camacho leg., P. Lo Cascio \& M. Romano det. (PLCC) (quoted in Lo Cascio \& Romano, 2004).

Distribution. West Mediterranean.

REMARKS. Recorded for Lipari and Panarea by Lo Cascio \& Romano (2004), quoted in Lo Cascio (2015)

\section{Tropidotilla litoralis (Petagna, 1787)}

EXAMIned MATERIAL. Lipari: 1 female, M.G. Di Palma leg., P. Lo Cascio \& M. Romano det. (MRC) (this material is quoted in Lo Cascio \& Romano, 2004).

DistribUtion. European Mediterranean.

REMARKS. Recorded for Lipari by Lo Cascio et al., (1998), Lo Cascio \& Romano (2004) and for Lipari and Vulcano by Lo Cascio (2015).

Nemka viduata ssp. viduata (Pallas, 1773)

EXAMINED MATERIAL. Lipari: Quattropani, 7. VI. 1997, 1 female, G.F. Turrisi leg. and det. (GFTC) 
(quoted in Turrisi, 1999); Cappero, VII.1995, 1 female, P. Lo Cascio leg., P. Lo Cascio \& M. Romano det. (PLCC); Palmeto, 1 female, P. Lo Cascio \& V. Pancioli leg., P. Lo Cascio \& M. Romano det. (PLCC); Vallone dei Lacci, VIII.1995, 1 female, P. Lo Cascio \& V. Pancioli leg., P. Lo Cascio \& M. Romano det. (PLCC); Timpone Ricotta, 8.X.1996, 3.VI.1997, 3 females, P. Lo Cascio leg., P. Lo Cascio \& M. Romano det. (PLCC). Vulcano: 1.VI.1956, 1 female, E. Di Napoli leg., P. Lo Cascio \& M. Romano det. (RAC); 3-17.VII.1993, 1 female, M. Mei leg., P. Lo Cascio \& M. Romano det. (MMC); Gran Cratere, 4.VI.1997, 4 females, P. Lo Cascio leg., P. Lo Cascio \& M. Romano det. (PLCC, MRC) (this material is quoted in Lo Cascio \& Romano, 2004).

Distribution. South European.

REMARKS. Recorded for Lipari by Turrisi (1999), for Lipari and Vulcano by Lo Cascio \& Romano (2004) and for Stromboli and the above mentioned islands by Lo Cascio (2015).

\section{Physetopoda pusilla (Klug, 1835)}

EXAMINED MATERIAL. Lipari: Vallone Muria, 18.VII.1996, 13.VIII.1996, 3 females, P. Lo Cascio leg., P. Lo Cascio \& M. Romano det. (PLCC); Fontanelle, 19.VIII.1996, 1 female, P. Lo Cascio \& S. Pasta leg., P. Lo Cascio \& M. Romano det. (PLCC) (this material is quoted in Lo Cascio \& Romano, 2004).

Distribution. South European.

REMARKS. Recorded for Lipari by Lo Cascio \& Romano (2004), quoted in Lo Cascio (2015).

\section{Smicromyrme ausonia Invrea, 1950}

EXAMINED MATERIAL. Lipari: Vallone Muria, 13.VIII.1996, 1 male, P. Lo Cascio leg., P. Lo Cascio \& M. Romano det. (PLCC); Castellaro, 21.VIII.1999, 1 male, P. Lo Cascio \& C. Moreno Camacho leg., P. Lo Cascio \& M. Romano det. (PLCC) (this material is quoted in Lo Cascio \& Romano, 2004).

Distribution. South European.

REMARKS. Recorded for Lipari by Lo Cascio \& Romano (2004), quoted in Lo Cascio (2015).

\section{Smicromyrme ruficollis (Fabricius, 1794)}

EXAMINED MATERIAL. Lipari: S. Lucia, VIII. 1996, 1 male (light trap), P. Lo Cascio leg., P. Lo Cascio \& M. Romano det. (PLCC); Fontanelle, 19.VIII.1996, 1 female, P. Lo Cascio \& S. Pasta leg., P. Lo Cascio \& M. Romano det. (PLCC); Vallone Muria, VIII-IX.1998, 7 males, P. Lo Cascio leg., P. Lo Cascio \& M. Romano det. (PLCC). Vulcano: 3-17.VII.1993, 1 female, M. Mei leg., P. Lo Cascio \& M. Romano det. (MMC); Monte Lentia, 4.VI.1997, 1 female, P. Lo Cascio leg., P. Lo Cascio \& M. Romano det. (PLCC). Stromboli: Scari, 24.VI.1998, 1 male (light trap), B. Carletti et al., leg., P. Lo Cascio \& M. Romano det. (PLCC) (this material is quoted in Lo Cascio \& Romano, 2004).

\section{DisTRIBUTION. European.}

REMARKS. Recorded for Lipari, Vulcano and Stromboli by Lo Cascio \& Romano (2004), quoted in Lo Cascio (2015).

Smicromyrme sulcisia Invrea, 1955

EXAMINED MATERIAL. Lipari: Cappero, VIII. 1995, 1 female, P. Lo Cascio leg., P. Lo Cascio \& M. Romano det. (PLC); Vallone Muria, VIIVIII.1996, 3.VI.1997, 1 male, 7 females, P. Lo Cascio leg., P. Lo Cascio \& M. Romano det. (PLC). Vulcano: Monte Lentia, 4.VI.1997, 1 male, 4 females, P. Lo Cascio leg., P. Lo Cascio \& M. Romano det. (PLC); Gran Cratere, 4.VI.1997, 22.VI.1998, 3 females, P. Lo Cascio et al. leg., P. Lo Cascio \& M. Romano det. (PLC); Piano, 23.V.2000, 1 female, P. Lo Cascio and C. Moreno Camacho leg., P. Lo Cascio \& M. Romano det. (PLC) (quoted in Lo Cascio \& Romano, 2004).

Distribution. South European.

REMARKS. Recorded for Lipari and Vulcano by Lo Cascio \& Romano (2004) as S. ingauna Invrea, 1958 (see also Lo Cascio, 2015).

Smicromyrme trinotata (A. Costa, 1858)

EXAMINED MATERIAL. Lipari: Vallone Muria, VII. 1995, 1 male, P. Lo Cascio leg. and det. (PLC) (quoted in Lo Cascio \& Romano, 2004).

Distribution. Sicilian Sardinian Corsican. 
REMARKS. Recorded for Lipari by Lo Cascio \& Romano (2004) as S. prope melanolepis (Costa, 1884) (see also Lo Cascio 2015).

Smicromyrme partita (Klug, 1835)

EXAMINED MATERIAL. Lipari: Vallone Muria, VII.1995, VII-VIII.1996, 14.X.1996, 3.VI.1997, 9 female, P. Lo Cascio leg. and det. (PLC) (quoted in Lo Cascio \& Romano, 2004).

Distribution. Mediterranean.

REMARKS. The species is recorded for Lipari as Smicromyrme partita sensu lato by Lo Cascio \& Romano (2004) due to the lack of males, hence the identification needs further study for confirmation.

Dasylabris maura ssp. maura (Linnaeus, 1758)

EXAMINED MATERIAL. Lipari: Timpone Ricotta, 4.X.1997, 27.V.1998, 20.IV.1998, 1 male, 3 females, P. Lo Cascio leg. and det. (PLC) (quoted in Lo Cascio \& Romano, 2004).

DisTRIBUTION. European.

REMARKS. Recorded for Lipari by Lo Cascio \& Romano (2004), quoted in Lo Cascio (2015).

\section{POMPILIDAE}

Pompilus cinereus Fabricius, 1798 (Fig. 11)

EXAMINED MATERIAL. Vulcano: Monte Lentia, 4.VI.1997, 1 female, P. Lo Cascio leg., on sand, G.F. Turrisi det. (PLCC). Vulcano: Piano, $330 \mathrm{~m}$ a.s.1., 9-22.VIII.2015, 2 males, G. Altadonna leg., yellow-pan trap, G.F. Turrisi det. (GAC).

Distribution. European Maghrebian.

Remarks. New for the Aeolian Archipelago.

Agenioideus ciliatus (Lepelletier, 1845)

EXAMINED MATERIAL. Lipari: Timpone Ricotta, 27.V.1998, 1 female, P. Lo Cascio leg., G.F. Turrisi det. (PLCC).

DisTRIBUTION. European Anatolian.

REMARKs. New for the Aeolian Archipelago.
Anoplius viaticus (Linnaeus, 1758)

EXAMINED MATERIAL. Lipari: Quattropani, 6. VI.1997, 4 males, G.F. Turrisi leg. and det. (GFTC).

Distribution. West Palaearctic.

REMARKs. New for the Aeolian Archipelago.

Aporus bicolor Spinola, 1808 (Fig. 12)

EXAMINED MATERIAL. Lipari: Urnazzo, 30. VII. 1996, 1 female, P. Lo Cascio leg., G.F. Turrisi det. (PLCC). Lipari: Quattropani, 6.VI.1997, 1 female, G.F. Turrisi leg. and det. (GFTC).

DisTRIBUTION. European Maghrebian Anatolian.

REMARKS. New for the Aeolian Archipelago.

Auplopus carbonarius (Scopoli, 1763)

EXAmined MATERIAL. Lipari: Pianoconte, 7. VI.1997, 1 male, G.F. Turrisi leg. and det. (GFTC). Lipari: Castello environs, 8.VI.1997, $1 \mathrm{f}$ emale, G.F. Turrisi leg. and det. (GFTC).

DistRibution. Palaearctic (introduced in North America).

REMARKS. New for the Aeolian Archipelago.

Priocnemis (Priocnemis) propinqua (Lepelletier, 1845)

EXAmined Material. Lipari: Pianoconte, 7. VI.1997, 1 female, G.F. Turrisi leg.-det. (GFTC).

DisTRIBUTION. Mediterranean.

REMARKS. New for the Aeolian Archipelago.

\section{VESPIDAE}

Leptochilus (Neoleptochilus) regulus (Saussure, 1855)

EXAMINED MATERIAL. Vulcano: 3-17.VII.1993, 1 male, M. Mei leg., W. Borsato det. (WBC).

Distribution. West Palaearctic.

REMARKS. The material examined is quoted in Borsato \& Turrisi (2004). 
Stenodynerus fastidiosissimus ssp. fastidiosissimus (Saussure, 1855)

EXAMINED MATERIAL. Vulcano: 3-17.VII.1993, 1 male, M. Mei leg., W. Borsato det. (WBC).

Distribution. West Palaearctic.

REMARKS. The material examined is quoted in Borsato \& Turrisi (2004).

Ancistrocerus auctus ssp. auctus (Fabricius, 1793)

EXAMINED MATERIAL. Lipari, 8.VI.1997, 1 male, G.F. Turrisi leg. (GFTC).

Distribution. European Asian.

REMARKS. The material examined is quoted in Borsato \& Turrisi (2004).

Ancistrocerus gazella (Panzer, 1798)

EXAMINED MATERIAL. Vulcano: VII.1993, 1 male, M. Mei leg., W. Borsato det. (WBC).

Distribution. Palaearctic (introduced in North America and New Zealand).

REMARKS. The material examined is quoted in Borsato \& Turrisi (2004).

Ancistrocerus longispinosus $\mathrm{ssp}$. longispinosus (Saussure, 1855)

EXAMINED MATERIAL. Lipari: main town, 8. VI.1997, 1 female, G.F. Turrisi leg., W. Borsato det. (GFTC) (quoted in Borsato \& Turrisi, 2004). Salina, 7.IV.1990, 1 female, M. Mei leg., W. Borsato det. (WBC) (quoted in Borsato \& Turrisi, 2004). Vulcano: VII.1993, 1 female, M. Mei leg., W. Borsato det. (WBC) (quoted in Borsato \& Turrisi, 2004); Piano, $330 \mathrm{~m}$ a.s.1., 2.VI.2012, 1 specimen, G. Altadonna leg., M. Selis det. (MSC); ibidem, 9.VIII.2013, 1 specimen, G. Altadonna leg., M. Selis det. (GAC); ibidem, 16.VIII.2014, 1 specimen, G. Altadonna leg., M. Selis det. (GAC); ibidem, 19.VIII.2014, 1 specimen, G. Altadonna leg., yellow-pan trap, M. Selis det. (MSC); ibidem, 22.VIII.2014, 1 specimen, G. Altadonna leg., yellow-pan trap, M. Selis det. (MSC).

DisTRIBUTION. Mediterranean.
REMARKS. Previously recorded for Vulcano, LIpari and Salina by Borsato \& Turrisi (2004).

Eumenes coarctatus ssp. maroccanus Gusenleitner, 1972

EXAMINED MATERIAL. Vulcano: VII.1993, 1 male, M. Mei leg., W. Borsato det. (WBC).

Distribution. South European Maghrebian.

REMARKS. The material examined is quoted in Borsato \& Turrisi (2004).

\section{Eumenes mediterraneus ssp. mediterraneus Kriechbaumer, 1879}

EXAmined material. Lipari: Pianoconte, 7. VI.1997, 1 male, 3 females, G.F. Turrisi leg., W. Borsato det. (GFTC) (quoted in Borsato \& Turrisi, 2004). Vulcano: Piano, 330 m a.s.1., 9-22.VIII.2015, 2 females, G. Altadonna leg., yellow-pan trap, M. Selis det. (MSC).

DistRIBUTION. Mediterranean.

REMARKS. Recorded for Lipari by Borsato \& Turrisi (2004), newly recorded for Vulcano.

Delta unguiculatum (Villers, 1789) (Fig. 13)

EXAMINED MATERIAL. Lipari: Monte Guardia, 2.VI.1997, 1 male, P. Lo Cascio leg., G.F. Turrisi det. (PLCC).

Distribution. South European West Asian.

REMARKS. New for the Aeolian Archipelago.

Rynchium oculatum (Fabricius, 1781)

EXAMINED MATERIAL. Vulcano: crater, VII.2017, 1 male, P. Lo Cascio leg., G.F. Turrisi det. (PLCC).

DisTRIBUTION. Mediterranean South West Asian.

REMARKS. New for the Aeolian Archipelago.

Vespa crabro ssp. crabro Linnaeus, 1758

Examined Material. Vulcano: Piano, $330 \mathrm{~m}$ a.s.1., 9.VIII.2015, 1 male, G. Altadonna leg., wine trap, M. Selis det. (GAC). 
Distribution. European Asian. This species is widely introduced in the Nearctic and Southern Hemisphere.

REMARKs. New for the Aeolian Archipelago.

\section{Polistes (Polistes) dominula (Christ, 1791)}

EXAmined MATERial. Vulcano: Piano, $330 \mathrm{~m}$ a.s.1., 21.VIII.2014, 1 male, G. Altadonna leg., M. Selis det. (GAC); ibidem, 9-22.VIII.2015, 1male, G. Altadonna leg., yellow-pan trap, M. Selis det. (MSC).

Distribution. European Maghrebian (introduced to South Africa, Australia and America).

REMARKS. New for the Aeolian Archipelago. The taxonomic concept and distribution of the species belonging to the genus Polistes Latreille herein identified follow the recent review of Schmid-Egger et al., (2017).

Polistes (Polistes) gallicus (Linnaeus, 1767) (Fig. 14)

EXAMINED MATERIAL. Panarea, 23.VI.1967, 2 females, Sulfaro leg., M. Selis det. (GFTC). Vulcano, 12.IV.1968, 1 female, G. Marcuzzi leg., M. Selis det. (GFTC); Vulcano: Capo Secco, 16.VIII.2015, 2 females, G. Altadonna leg., M. Selis det. (GAC); Vulcano: Piano, 330 m a.s.1., 19.VIII.2014, 1 specimen, G. Altadonna leg., yellow-pan trap, M. Selis det. (MSC); Piano, 9-22.VIII.2015, 9 females, 2 males, G. Altadonna leg., yellow-pan trap, M. Selis det. (MSC); ibidem, 12-18.VIII.2016, 5 specimens, G. Altadonna leg., yellow-pan trap, M. Selis det. (MSC).

Distribution. Mediterranean.

Remarks. New for the Aeolian Archipelago.

\section{Polistes (Polistes) nimpha (Christ, 1791)}

EXAmined MATERial. Vulcano: Piano, $330 \mathrm{~m}$ a.s.1., 9-22.VIII.2015, 1 female, G. Altadonna leg., yellow pan trap, M. Selis det. (MSC); ibidem, 1218.VIII.2016, yellow pan trap, 2 specimens, G. Altadonna leg., M. Selis det. (MSC).

Distribution. European Asian.

REMARKS. New for the Aeolian Archipelago.
Vespula (Paravespula) germanica (Fabricius, 1793)

EXAMINED MATERIAL. Vulcano: Gelso, 30. VIII.2014, 1 specimen, G. Altadonna leg., M. Selis det. (MSC); Vulcano: Piano, 3.III.2019, 1 specimen, G. Altadonna leg., G.F. Turrisi det. (GAC). Lipari: Canneto, 21.II.1966, 1 female, M. La Greca leg., G.F. Turrisi det. (GFTC).

DistriBution. Palaearctic (largely introduced and widespread in the Nearctic).

REMARKS. New for the Aeolian Archipelago.

\section{SPHECIDAE}

Sceliphron destillatorium (Illiger, 1807)

EXAmined MATERIAL. Panarea, 18.VI.1967, 1 female, Sulfaro leg., G.F. Turrisi det. (GFTC).

Distribution. South Palaearctic.

REMARKS. Recorded for Panarea by Turrisi \& Altadonna (2017).

Sceliphron spirifex (Linnaeus, 1758)

EXAMINED MATERIAL. Vulcano: Piano, $330 \mathrm{~m}$ a.s.1., 6.VII.2012, 1 female, G. Altadonna leg., G.F. Turrisi det. (GFTC).

Distribution. West Palaearctic.

REMARKS. Recorded for Vulcano by Turrisi \& Altadonna (2017), already known for other circumsicilian islands (Ustica and Lampedusa) (Riggio \& De Stefani Perez, 1888; Pagliano \& Scaramozzino, 1995).

\section{Ammophila heydeni Dahlbom, 1845}

EXAMINED MATERIAL. Lipari: Vallone Muria, 14. VII.1995, 1 female, P. Lo Cascio leg., G.F. Turrisi det. (PLCC).

Distribution. Mediterranean Asian.

REMARKs. New for the Aeolian Archipelago.

Prionyx subfuscatus (Dahlbom, 1845) (Fig. 15)

EXAMINED MATERIAL. Lipari: Timpone Ricotta, 
8.X.1996, 1 female, P. Lo Cascio leg., G.F. Turrisi det. (PLCC).

Distribution. Mediterranean Asian.

Remarks. New for the Aeolian Archipelago.

Prionyx lividocinctus (A. Costa, 1858)

Examined material. Lipari: Contrada San Giorgio, 15.X.1967, 1 male, Cirotti leg., G.F. Turrisi det. (GFTC).

Distribution. Mediterranean Asian.

Remarks. New for the Aeolian Archipelago.

Sphex funerarius Gussakovskij, 1934

ExAmined MATERIAL. Lipari: Monte Chirica, 26.IX.1996, 1 male, P. Lo Cascio leg., G.F. Turrisi det. (PLCC); Lipari: Madoro, 21.VIII.1996, 1 male, P. Lo Cascio \& S. Pasta leg., G.F. Turrisi det. (PLCC).

Distribution. Mediterranean Asian.

Remarks. New for the Aeolian Archipelago.

\section{CRABRONIDAE}

Dryudella esterinae Pagliano, 2001 (Fig. 16)

EXAMINED MATERIAL. Lipari: Vallone Muria, 3. VI.1997, 1 male, P. Lo Cascio leg., G.F. Turrisi det. (PLCC).

Distribution. South West European.

REMARKS. New for the Aeolian Archipelago and Sicily.

Dryudella tricolor (Van der Linden, 1829)

EXAMINED MATERIAL. Lipari: Pianoconte, 7. VI. 1997, 1 female, G.F. Turrisi leg. and det. (GFTC).

Distribution. European Asian.

RemarKs. New for the Aeolian Archipelago.

Harpactus laevis (Latreille, 1792)

EXAMINED MATERIAL. Lipari: Vallone Muria,
VII.1999, 1 male, P. Lo Cascio leg., Moericke trap, G.F. Turrisi det. (PLCC).

Distribution. European Asian.

REMARKS. New for the Aeolian Archipelago.

Nysson quadriguttatus Spinola, 1808

EXAmined MATERIAL. Vulcano: Piano, 9.VI. 20 13, 1 female, G. Altadonna leg., yellow pan trap, G.F. Turrisi det. (GAC).

DistriBution. European.

REMARKS. New for the Aeolian Archipelago and Sicily.

Crossocerus distinguendus (Morawitz, 1866)

EXAMINED MATERIAL. Lipari: Castello environs, 8.VI.1997, 1 male, G.F. Turrisi leg. et det. (GFTC). Lipari: Pianoconte, 7.VI.1997, 1 male, G.F. Turrisi leg. et det. (GFTC).

DistRIBUTION. European Maghrebian.

REMARKS. New for the Aeolian Archipelago.

Crossocerus quadrimaculatus (Fabricius, 1793)

EXAMINED MATERIAL. Lipari: Pianoconte, 7.VI. 1997, 1 male, G.F. Turrisi leg. et det. (GFTC).

DisTRIBUTION. Palaearctic.

REMARKS. New for the Aeolian Archipelago.

Lestica clypeata (Schreber, 1759) (Fig. 17)

EXAMINED MATERIAL. Lipari: Urnazzo, 2.VI. 1997, 1 male, P. Lo Cascio leg., on Daucus sp., G.F. Turrisi det. (PLCC).

Distribution. Palaearctic.

REMARKs. New for the Aeolian Archipelago.

Tracheliodes quinquenotatus (Jurine,1807) (Fig. 18)

EXAmined Material. Lipari: Pianoconte, 7.VI. 1997, 1 male, G.F. Turrisi leg. et det. (GFTC). Vulcano: Piano, 330 m a.s.1., 9-22.VIII.2015, 2 males, G. Altadonna leg., yellow pan trap, G.F. Turrisi det. (GAC). 


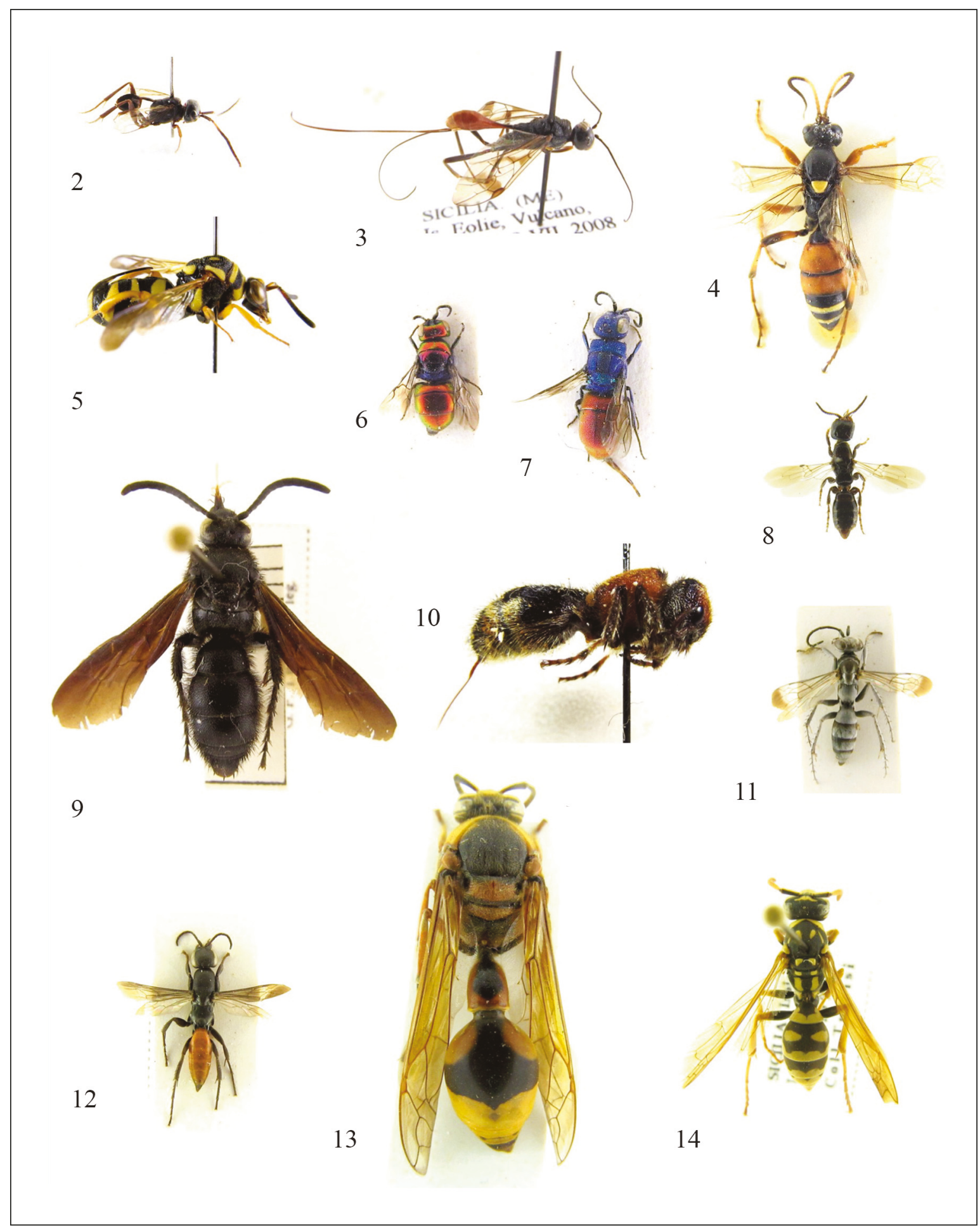

Figures 2-14. Hymenoptera of Aeolian Archipelago (not in scale). Fig. 2: Zeuxevania splendidula female (Lipari). Fig. 3: Pristaulacus lindae female (Vulcano). Fig. 4: Eutanyacra picta female (Vulcano). Fig. 5: Leucospis gigas female (Lipari). Fig. 6: Holopyga fervida female (Lipari). Fig. 7: Chrysis cerastes female (Salina). Fig. 8: Meria dorsalis female (Lipari). Fig. 9: Scolia carbonaria male (Lipari). Fig. 10: Ronisia brutia female (Lipari). Fig. 11: Pompilus cinereus female (Vulcano). Fig. 12: Aporus bicolor female (Lipari). Fig. 13: Delta unguiculatum male (Lipari). Fig. 14: Polistes gallicus worker (Lipari). 
Distribution. Mediterranean.

REMARKS. New for the Aeolian Archipelago.

Tachysphex costae (De Stefani-Perez, 1882)

Examined Material. Vulcano: Piano, 9.VI. 2013, 1 female, G. Altadonna leg., yellow pan trap, G.F. Turrisi det. (GAC).

Distribution. Southern West Palaearctic.

Remarks. New for the Aeolian Archipelago.

Tachysphex nitidior Beaumont, 1940

EXAmined Material. Vulcano: Piano, 9.VI. 2013, 1 male, G. Altadonna leg., yellow pan trap, G.F. Turrisi det. (GAC).

Distribution. Southern West Palaearctic.

REMARKS. New for the Aeolian Archipelago.

Miscophus eatoni Saunders, 1903

Examined material. Vulcano: Piano, $330 \mathrm{~m}$ a.s.1., 9-22.VIII.2015, 1 female, G. Altadonna leg., yellow pan trap, M. Selis det. (MSC).

Distribution. Mediterranean.

REMARKS. New for the Aeolian Archipelago and Sicily.

Miscophus lusitanicus Andrade, 1952

EXAmined MATERIAL. Panarea: Soldata, $230 \mathrm{~m}$ a.s.1., 21.VIII.2015, 1 male, G. Altadonna leg., yellow pan trap, G.F. Turrisi det. (GAC).

Distribution. South European Anatolian.

Remarks. New for the Aeolian Archipelago; first record for Italy.

\section{Miscophus pretiosus Kohl, 1884}

Examined material. Vulcano: Piano, $330 \mathrm{~m}$ a.s.1., 9-22.VIII.2015, 1 female, G. Altadonna leg., yellow-pan trap, G.F. Turrisi det. (GAC).

Distribution. South European Anatolian.

REMARKs. New for the Aeolian Archipelago.

\section{Solierella compedita (Piccioli, 1869)}

EXAMINED MATERIAL. Lipari: Vallone Muria, VII.1999, 1 male, P. Lo Cascio leg., Moericke trap, G.F. Turrisi det. (PLCC). Panarea: Soldata, $230 \mathrm{~m}$ a.s.1., 21.VIII.2015, 2 males, G. Altadonna leg., yellow-pan trap, M. Selis det. (MSC); ibidem, 21.VIII.2015, 2 males, G. Altadonna leg., yellowpan trap, G.F. Turrisi det. (GAC).

Distribution. West Palaearctic.

REMARKS. New for the Aeolian Archipelago.

Pison atrum (Spinola, 1808)

EXAMINED MATERIAL. Lipari: Castello environs, 8.VI.1997, 1 female, G.F. Turrisi leg. and det. (GFTC).

Distribution. Mediterranean.

REMARKS. New for the Aeolian Archipelago.

Trypoxylon kolazyi Kohl, 1893

EXAMINED MATERIAL. Lipari: Castello environs, 8.VI.1997, 2 males, G.F. Turrisi leg. and det. (GFTC). Vulcano: Piano, $330 \mathrm{~m}$ a.s.1., 922.VIII.2015, 1 female, G. Altadonna leg., yellow pan trap, G.F. Turrisi det. (GAC).

Distribution. South European Anatolian.

REMARKS. New for the Aeolian Archipelago. Recently recorded for Sicily (Schmid-Egger, 2003; Tomarchio \& Turrisi, 2006) and Lampedusa (Pagliano, 2003).

\section{Trypoxylon deceptorium Antropov, 1991}

EXAmined Material. Vulcano: Piano, $330 \mathrm{~m}$ a.s.1., 9-22.VIII.2015, 1 female, G. Altadonna leg., yellow pan trap, M. Selis det. (MSC).

Distribution. European South West Asian.

Remarks. New for the Aeolian Archipelago. Recently recorded for Sicily by Tomarchio \& Turrisi (2006).

Trypoxylon scutatum Chevrier, 1867

EXAMINED MATERIAL. Lipari: Castello environs, 
8.VI.1997, 1 male, G.F. Turrisi leg. and det. (GFTC). Vulcano: Piano, $330 \mathrm{~m}$ a.s.1., 922.VIII.2015, 3 females, G. Altadonna leg., yellow pan trap, M. Selis det. (GAC); ibidem, 1218.VIII.2016, 2 specimens, G. Altadonna leg., yellow pan trap, M. Selis det. (MSC).

DisTRIBUTION. European Anatolian.

REMARKs. New for the Aeolian Archipelago.

Ammoplanus perrisi Giraud, 1869

EXAMINED MATERIAL. Lipari: Castello environs, 8.VI.1997, 1 male, G.F. Turrisi leg. and det. (GFTC).

Distribution. European.

Remarks. New for the Aeolian Archipelago.

Passaloecus pictus Ribaut, 1952

EXAmined MATERIAL. Lipari: Pianoconte, 7.VI. 1997, 2 females, G.F. Turrisi leg. and det. (GFTC). Lipari: Castello environs, 8.VI.1997, 7 females, G.F. Turrisi leg. and det. (GFTC).

Distribution. South European Anatolian.

REMARKS. New for the Aeolian Archipelago. Recorded for Sicily by Schmid-Egger (2003) and Tomarchio \& Turrisi (2006).

Pemphredon austriaca (Kohl, 1888)

Examined material. Lipari: Pianoconte, 7.VI. 1997, 1 male, G.F. Turrisi leg. and det. (GFTC).

DisTRIBUTION. European.

Remarks. New for the Aeolian Archipelago.

Mimesa grandii Maidl, 1933 (Fig. 19)

EXAMINED MATERIAL. Lipari: Castello environs, 8.VI.1997, 1 female, G.F. Turrisi leg. et det. (GFTC).

Distribution. West Palaearctic.

Remarks. New for the Aeolian Archipelago.

Cerceris circularis ssp. dacica Schletterer, 1887

EXAMINED MATERIAL. Lipari: Castello environs,
8.VI.1997, 1 male, G.F. Turrisi leg. et det., on Daucus sp. (GFTC).

DisTRIBUTION. South European.

REMARKS. New for the Aeolian Archipelago and Sicily.

Philanthus coarctatus ssp. siculus Giordani Soika, 1944 (Fig. 20)

EXAMINED MATERIAL. Lipari: Castello environs, 8.VI.1997, 4 males, G.F. Turrisi leg. et det., on Daucus sp. (GFTC).

Distribution. South Italian.

REMARKS. New for the Aeolian Archipelago.

\section{FORMICIDAE}

Aphaenogaster pallida (Nylander, 1848)

EXAmined MATERIAL. Filicudi: Fossa delle Felci, 1.I.1997, P. Lo Cascio leg., F. Rigato det. (PLCC). Lisca Bianca: 7.IV.1996, P. Lo Cascio \& V. Pancioli leg., F. Rigato det. (PLCC).

Distribution. South East European.

REMARKS. Recorded for Lipari (Kutter, 1927) and Salina (Schär et al., 2020), is newly recorded for Filicudi and the islet Lisca Bianca.

Aphaenogaster subterranea (Latreille, 1798)

EXAMINED MATERIAL. Strombolicchio: 29. VI.1998, P. Lo Cascio leg., F. Rigato det. (PLCC).

Distribution. Mediterranean European.

REMARKS. Recorded for all the main islands except Vulcano (Schär et al., 2020), is newly recorded for the islet Strombolicchio.

Camponotus lateralis (Olivier, 1791)

EXAMINED MATERIAL. Vulcano: Monte Lentia, 4.VI.1997, P. Lo Cascio leg., F. Rigato det. (PLCC).

Distribution. Mediterranean Turanian.

REMARKS. Recorded for Lipari (Kutter, 1927), 
Vulcano (Baroni Urbani, 1964), Salina, Stromboli, Filicudi and Panarea (Schär et al., 2020).

Camponotus micans (Nylander, 1856)

EXAMINED MATERIAL. Lipari: Timpone Ricotta, 8.X.1996, P. Lo Cascio leg., F. Rigato det. (PLCC).

Distribution. West Mediterranean.

REMARKS. Recorded for Lipari (Kutter, 1927), Salina, Vulcano and Filicudi (Schär et al., 2020).

\section{Camponotus nylanderi Emery, 1921}

EXAmined MATERIAL. Lipari: Timpone Ricotta, 8.X.1996, P. Lo Cascio leg., F. Rigato det. (PLCC). Filicudi: Fossa delle Felci, 1.I.1997, P. Lo Cascio leg., F. Rigato det. (PLCC). Alicudi: Pianicello, 2.IV.1997, P. Lo Cascio \& G. Serra leg., F. Rigato det. (PLCC). Strombolicchio: 6.VIII.1994, P. Lo Cascio \& V. Pancioli leg., F. Rigato det. (PLCC); 29.VI.1998, P. Lo Cascio leg., F. Rigato det. (PLCC). Scoglio Faraglione: 5.VIII.1994, P. Lo Cascio \& V. Pancioli leg., F. Rigato det. (PLCC).

Distribution. South Italian.

REMARKS. Recorded for Lipari (Kutter, 1927), the islet Strombolicchio (Lo Cascio, 2004), Salina, Vulcano, Stromboli, Filicudi and Panarea (Schär et al., 2020), is newly recorded for Alicudi and the islet Scoglio Faraglione.

Crematogaster scutellaris (Olivier, 1791)

EXAMINED MATERIAL. Lipari: Vallone Muria, 14. X.1998, P. Lo Cascio leg., F. Rigato det. (PLCC).

Distribution. Mediterranean.

REMARKS. Recorded for Lipari (Kutter, 1927), Salina (Monastero \& Genduso, 1964b), Vulcano, Stromboli, Filicudi and Panarea (Schär et al., 2020).

Lepisiota nigra (Dalla Torre, 1893)

EXAmined Material. Strombolicchio: 25.VI. 1998, P. Lo Cascio leg., F. Rigato det. (PLCC).

DistRIBUTION. Mediterranean Turanian.

REMARKS. Recorded for Lipari, Salina, Vulcano and Panarea (Schär et al., 2020), is newly recorded for the islet Strombolicchio.

Messor capitatus (Latreille, 1798)

EXAMINED MATERIAL. Lipari: Timpone Ricotta, 8.X.1996, P. Lo Cascio leg., F. Rigato det. (PLCC); Fuardo, 9.X.1996, P. Lo Cascio leg., F. Rigato det. (PLCC).

Distribution. West Mediterranean.

REMARKS. Recorded for Lipari (Kutter, 1927; Baccetti, 1967; Schär et al., 2020).

Messor minor ssp. calabricus Santschi, 1927

EXAMINED MATERIAL. Lisca Bianca: 7.IV.1996, P. Lo Cascio \& V. Pancioli leg., F. Rigato det. (PLCC).

DiSTRIBUTION. Southern Italian.

REMARKS. Recorded for Lisca Bianca (Lo Cascio \& Navarra, 2003).

Messor structor (Latreille, 1798)

EXAMINED MATERIAL. Lipari: Fuardo, 9.X.1996, P. Lo Cascio leg., F. Rigato det. (PLCC).

Distribution. South East European.

REMARKS. Already recorded for Lipari (Kutter, 1927).

Pheidole pallidula (Nylander, 1848)

ExAmined MATERIAL. Stromboli: path to the crater, 400 m a.s.1., 24.X.1996, P. Lo Cascio leg., F. Rigato det. (PLCC).

DisTRIBUTION. West Mediterranean.

REMARKS. Recorded for Lipari (Kutter, 1927), Filicudi (Seifert, 2016), Salina, Vulcano, Stromboli and Panarea (Schär et al., 2020).

Plagiolepis pallescens Lomnicki, 1925

EXAMINED MATERIAL. Strombolicchio: 6.IV.1996, P. Lo Cascio \& V. Pancioli leg., F. Rigato det. (PLCC).

Distribution. West Palaearctic. 
REMARKS. Recorded for all the main islands except Filicudi (Schär et al., 2020), is newly recorded for the islet Strombolicchio.

Ponera coarctata (Latreille, 1802)

Examined material. Alicudi: Pianicello, 2.IV.1997, P. Lo Cascio \& G. Serra leg., F. Rigato det. (PLCC).

Distribution. South European.

REMARKS. Recorded for Alicudi (Lo Cascio et al., 2006), Lipari and Salina (Schär et al., 2020).

Tetramorium punctatum Santschi, 1927

Examined material. Scoglio Faraglione: 6 . VIII.1994, P. Lo Cascio \& V. Pancioli leg., F. Rigato det. (PLCC).

DisTRIBUTION. East Mediterranean.

REMARKS. Recorded for Lipari (Kutter, 1927 as T. semilaeve; Santschi, 1927 as T. semilaeve var. $l i-$ pareum nov.; Sanetra et al. 1999), Salina, Vulcano, Stromboli, Filicudi and Panarea (Schär et al., 2020), is newly recorded for the islet Scoglio Faraglione.

\section{COLLETIDAE}

\section{Colletes nigricans Gistel, 1857}

Material examined. Salina, Monte dei Porri, 20.IX.1966, 2 females, Arcidiacono leg., V. Nobile det. (VNC).

Distribution. West Mediterranean.

Remarks. New for the Aeolian Archipelago.

Hylaeus (Paraprosopis) clypearis (Schenck, 1853)

Examined Material. Panarea: 23.VI.1967, 1 female, Sulfaro leg., V. Nobile det. (VNC).

Distribution. South West Palaearctic (introduced in Chile).

REMARKS. New for the Aeolian Archipelago.

\section{Hylaeus (Dentigera) imparilis Förster, 1871}

EXAMINED MATERIAL. Lipari: Chiesa Vecchia, 7.VII.1999, 1 female, G.F. Turrisi leg., V. Nobile det., on cultivated Petroselimum sp. (GFTC).

Distribution. South West Palaearctic (introduced in Chile).

REMARKS. New for the Aeolian Archipelago.

Hylaeus (Spatulariella) punctatus (Brullé, 1832)

Examined material. Salina: Monte dei Porri, 20.IX.1966, 1 female, Arcidiacono leg., V. Nobile det. (VNC).

Distribution. South West Palaearctic (introduced in Chile).

REMARKS. New for the Aeolian Archipelago.

\section{ANDRENIDAE}

\section{Andrena (Charitandrena) hattorfiana ssp. dimidiata Brullé, 1832}

EXAMINED MATERIAL. Vulcano: 29.V-4.VI.2010, 1 male, M. Mei leg., V. Nobile det. (VNC).

Distribution. Central South Anatolian European. The nominate subspecies has a West European Asian distribution.

REMARKs. New for the Aeolian Archipelago.

Andrena (Euandrena) ruficrus Nylander, 1848

EXAmined MATERIAL. Lipari: Acquacalda, 29. IV.1966, 1 female, I. Marcellino leg., V. Nobile det. (VNC).

Distribution. European West Asian.

REMARKS. New for the Aeolian Archipelago.

Andrena (Melandrena) morio ssp. morio Brullé, 1832

EXAMINED MATERIAL. Vulcano: 26.IV-4.V.2009, 2 females, M. Mei leg., V. Nobile det. (VNC); Panarea: Drauto, 20 m a.s.1., 21.VIII.2015, G. Altadonna leg., 1 female, G.F. Turrisi det. (GAC). 
Distribution. South East European West Asian.

REMARKS. New for the Aeolian Archipelago.

Andrena (Melandrena) nigroaenea ssp. nigroaenea (Kirby, 1802)

EXAmined MATERIAL. Filicudi, 1 male, M. Mei leg., V. Nobile det. (VNC).

Distribution. European Asian.

Remarks. New for the Aeolian Archipelago.

Andrena (Melandrena) nitida ssp. nitida (Müller, 1776)

EXAmined Material. Vulcano: Piano, 14. III.2000, 1 female, P. Lo Cascio \& S. Pasta leg., on flowers of Cytisus aeolicus (PLCC).

Distribution. European.

REMARKs. New for the Aeolian Archipelago.

Andrena (Plastandrena) pilipes Fabricius, 1781

EXAMINED MATERIAL. Vulcano, 29.V.4.VI.2010, 2 females, M. Mei leg., V. Nobile det. (VNC).

Distribution. Palaearctic.

REMARKS. New for the Aeolian Archipelago.

Andrena (Scitandrena) scita Eversmann, 1852

EXAMINED MATERIAL. Vulcano, 29.V-4.VI.2010, 1 male, M. Mei leg., V. Nobile det. (VNC).

Distribution. South East European South West Asian.

REMARKS. New for the Aeolian Archipelago.

Andrena (Taeniandrena) wilkella (Kirby, 1802)

EXAMINED MATERIAL. Vulcano, 25.IV-4.V.2009, 1 female, M. Mei leg., V. Nobile det. (VNC).

Distribution. European Asian.

Remarks. New for the Aeolian Archipelago.
Pseudapis bispinosa (Brullé, 1832)

EXAMINED MATERIAL. Vulcano: 3-17.VII.1993, 6 males, M. Mei leg., V. Nobile det. (VNC).

Distribution. Mediterranean Asian.

REMARKs. New for the Aeolian Archipelago.

Ceylalictus variegatus (Olivier, 1789)

EXAMINED MATERIAL. Vulcano: 24.VII.1963, 2 males, 1 female, V. Nobile leg. et det. (VNC). Vulcano: 3-17.VII.1993, 1 male, 1 female, M. Mei leg., V. Nobile det. (VNC).

Distribution. Mediterranean Asian.

REMARKS. New for the Aeolian Archipelago.

Nomioides facilis (Smith, 1853)

EXAMINED MATERIAL. Lipari: Urnazzo, 2.VI. 1997, 1 female, P. Lo Cascio leg., on Asteraceae, V. Nobile det. (PLCC).

Distribution. Mediterranean Asian.

REMARKS. New for the Aeolian Archipelago.

Nomioides minutissimus (Rossi, 1790)

EXAmined Material. Vulcano: Piano, 9-22. VIII.2015, 1 female, G. Altadonna leg., V. Nobile det. (yellow pan-trap) (GAC).

Distribution. Mediterranean South West Asian.

REMARKS. New for the Aeolian Archipelago.

Halictus (Halictus) quadricinctus (Fabricius, 1776)

EXAMINED MATERIAL. Panarea: 12.VI.1967, 1 female, V. Nobile leg. et det. (VNC). Vulcano: 28.VI18.VI.2008, 2 males, M. Mei leg., V. Nobile det. (VNC); 29.V-4.VI.2010, 1 female, M. Mei leg., V. Nobile det. (VNC).

Distribution. South Palaearctic.

REMARKS. New for the Aeolian Archipelago.

Halictus (Hexataenites) fulvipes (Klug, 1817) EXAMINED MATERIAL. Lipari: Monte Chirica,

\section{HALICTIDAE}


26.IX.1996, 1 male, P. Lo Cascio leg., V. Nobile det. (PLCC). Panarea: 18.VI.1967, 1 female, Sulfaro leg., V. Nobile det. (VNC). Vulcano: 24.VII.1963, 1 female, V. Nobile leg. et det. (VNC); 10.VII.1993, 2 males, M. Mei leg., V. Nobile det. (VNC).

Distribution. Mediterranean Asian (introduced in Mexico).

REMARKS. New for the Aeolian Archipelago.

Halictus (Hexataenites) scabiosae (Rossi, 1790) (Fig. 21)

EXAmined mATERial. Lipari: Vallone Muria, VII.1999, 1 female, P. Lo Cascio leg., V. Nobile det. (Moericke trap) (PLCC).

Distribution. South West Palaearctic (extended to South Africa).

Remarks. New for the Aeolian Archipelago.

Halictus (Monilapis) compressus Walckenaer, 1802

ExAmined material. Lipari: Quattropani, 6. VI.1997, 1 male, 2 females, on flowers of Rubus sp., G.F. Turrisi leg., V. Nobile det. (GFTC).

Distribution. South European Central Asian (excluding Iberian Peninsula).

REMARKS. New for the Aeolian Archipelago.

Halictus (Seladonia) gemmeus Dours, 1872

EXAMINED MATERIAL. Vulcano: 3-17.VII.1993, 1 male, M. Mei leg., V. Nobile det. (VNC).

Distribution. South Palaearctic.

REMARKS. New for the Aeolian Archipelago.

Halictus (Seladonia) smaragdulus Vachal, 1895

EXAMINED MATERIAL. Vulcano: 24.VII.1963, 2 females, V. Nobile leg. and det. (VNC).

Distribution. South West Palaearctic.

REMARKS. New for the Aeolian Archipelago.

Halictus (Tytthalictus) asperulus Perez, 1895

EXAmined MAterial. Panarea: 23.VI.1967, 1 male, Sulfaro leg., V. Nobile det. (VNC). Vulcano, 15.VII.1993, 1 male, M. Mei leg., V. Nobile det. (VNC).

Distribution. Mediterranean South West Asian.

REMARKS. New for the Aeolian Archipelago.

Halictus (Tythalictus) maculatus Smith, 1848

EXAMINed MATERIAL. Panarea: 23.VI.1967, 1male, Sulfaro leg., V. Nobile det. (VNC).

DistRIBUTION. South European Turanian.

REMARKS. New for the Aeolian Archipelago.

Lasioglossum (Dialictus) nitidulum (Fabricius, 1804)

EXAMINED MATERIAL. Lipari: Monte Sant'Angelo, $500 \mathrm{~m}$ a.s.1., 21.II.1966, 2 females, M. La Greca leg., V. Nobile det. (VNC).

Distribution. South European Central Asian.

REMARKS. New for the Aeolian Archipelago.

Lasioglossum (Dialictus) planulum (Perez, 1903)

EXAmined MATERial. Lipari: Pianoconte, 7. VI.1997, 1 male, G.F. Turrisi leg., V. Nobile det. (GFTC).

DistriBUTION. North Mediterranean.

REMARKS. New for the Aeolian Archipelago.

Lasioglossum (Dialictus) semilucens (Alfken, 1914)

EXAMINED MATERIAL. Lipari: Castello environs, 8.VI.1997, G.F. Turrisi leg., V. Nobile det. (GFTC).

Distribution. Central South European Central Asian.

REMARKS. New for the Aeolian Archipelago and Sicily.

\section{Lasioglossum (Sphecodogastra) immunitum} ssp. sillatum (Warncke, 1981)

EXAMINED MATERIAL. Vulcano: 3-17.VII.1993, 1 male, M. Mei leg., V. Nobile det. (VNC). 
Distribution. Sicilian Sardinian South Italian. The nominate subspecies has a Mediterranean Iranian distribution (discontinuous in the South East Mediterranean).

REMARKS. Described from Catania (holotype) and Lipari: Mount Guardia (paratypes) (Warncke, 1981). The holotype was collected on flowers of Centaurea (Asteraceae).

\section{Lasioglossum (Sphecodogastra) malachurum} (Kirby, 1802)

Examined material. Lipari: Mount Sant'Angelo, m 500, 21.II.1966, 1 female, M. La Greca leg. (DSCC); Pianoconte, 7.VI.1997, 1 male, G.F. Turrisi leg., V. Nobile det. (GFTC).

Distribution. Central South West Palaearctic Macaronesian.

REMARKs. New for the Aeolian Archipelago.

Lasioglossum (Sphecodogastra) opacum Perez, 1895

EXAMINED MATERIAL. Lipari: Quattropani-Acquacalda, 22.II.1966, 1 female, M. La Greca leg., V. Nobile det. (DSCC); Acquacalda, 29.VI.1966, 1 female, I. Marcellino leg., V. Nobile det. (DSCC); Urnazzo, 2.VI.1997, 1 male, P. Lo Cascio leg., on Asteraceae, V. Nobile det. (PLCC); Quattropani, 6.VI.1997, 1 male, G.F. Turrisi leg., V. Nobile det. (GFTC); Pianoconte, 7.VI.1997, 1 female, G.F. Turrisi leg., V. Nobile det. (GFTC). Vulcano: 10.VII.1993, 3 males, 2 females, M. Mei leg., V. Nobile det. (VNC).

DisTRIBUTION. Mediterranean Iranian.

REMARKS. New for the Aeolian Archipelago.

Lasioglossum (Sphecodogastra) nigripes (Lepelletier, 1841)

EXAmined MATERIAL. Lipari: Mendolita, 30. VI.1967, 1 female, Cirotti leg., V. Nobile det. (DSCC).

Distribution. Central South West Mediterranean Macaronesian.

Remarks. New for the Aeolian Archipelago.

Lasioglossum (Sphecodogastra) pauxillum (Schenck, 1853)

Examined MAterial. Panarea: 12.VI.1967, 2 males, V. Nobile leg. (VNC); 23.VI.1967, 1 males, Sulfaro leg., V. Nobile det. (DSCC).

Distribution. Central South West Palaearctic.

REMARKS. New for the Aeolian Archipelago.

\section{Lasioglossum (Sphecodogastra) subhirtum} (Lepelletier, 1841)

EXAMINED MATERIAL. Lipari: Urnazzo, 2. VI. 1997, 1 male, on flowers of Daucus sp. (Apiaceae), P. Lo Cascio leg., V. Nobile det. (LPC).

Distribution. Central South West Mediterranean.

REMARKS. New for the Aeolian Archipelago.

\section{Lasioglossum (Lasioglossum) albocinctum (Lucas, 1849)}

EXAMINED MATERIAL. Vulcano: 10.VII.1993, 3 males, 1 female, M. Mei leg., V. Nobile det. (VNC).

DisTRIBUTION. European Mediterranean (discontinuous in North East Africa).

REMARKS. New for the Aeolian Archipelago.

\section{Lasioglossum (Lasioglossum) bimaculatum}

(Dours, 1872)

EXAmined material. Lipari: Pianoconte, 24. V.1967, 1 female, V. Nobile leg. and det. (VNC). Vulcano: 3-17.VII.1993, 1 female, M. Mei leg., V. Nobile det. (VNC).

Distribution. West Mediterranean.

REMARKS. New for the Aeolian Archipelago.

Lasioglossum (Lasioglossum) costulatum (Kriechbaumer, 1873)

EXAMined MATERIAL. Lipari: Pianoconte, 24. V.1967, 1 female, V. Nobile leg. and det. (VNC).

DisTRIBUTION. Palaearctic.

REMARKS. New for the Aeolian Archipelago.

Lasioglossum (Lasioglossum) leucozonium ssp. cedri Ebmer, 1976 
EXAMINED MATERIAL. Lipari: Acquacalda, 29. IV.1966, 1 female, I. Marcellino leg., V. Nobile det. (DSCC).

Distribution. South West Mediterranean. The species is European Central Asian.

Remarks. New for the Aeolian Archipelago.

Sphecodes albilabris (Fabricius, 1793) (Fig. 22)

EXAMINED MATERIAL. Lipari: Vallone Muria, 3. VI.1997, 1 female, P. Lo Cascio leg., V. Nobile det. (PLCC).

Distribution. European Central Asian.

Remarks. New for the Aeolian Archipelago.

Sphecodes gibbus (Linnaeus, 1758)

EXAMINED MATERIAL. Vulcano: 25.IV-4.V.2009, 1 female, M. Mei leg., V. Nobile det. (VNC).

Distribution. European Asian.

Remarks. New for the Aeolian Archipelago.

Sphecodes monilicornis ssp. quadratus Meyer, 1919

ExAmined MATERIAL. Vulcano: 1male, M. Mei leg., V. Nobile det. (VNC).

Distribution. South European. The species is Palaearctic.

REMARKS. New for the Aeolian Archipelago.

Sphecodes spinulosus Hagens, 1875

EXAMINED MATERIAL. Vulcano: 25.IV-4.V.2009, 1 female, M. Mei leg., V. Nobile det. (VNC).

Distribution. European West Asian.

Remarks. New for the Aeolian Archipelago.

\section{MEGACHILIDAE}

Heriades (Heriades) truncorum (Linnaeus, 1758)

EXAMINED MATERIAL. Vulcano: 25.IV-4.V.2009, 1 male, M. Mei leg., V. Nobile det. (VNC).
Distribution. West Central Palaearctic.

REMARKS. New for the Aeolian Archipelago.

Hoplitis (Hoplitis) adunca ssp. adunca (Panzer, 1798)

EXAMINED MATERIAL. Vulcano: 25.IV-4.V.2009, 1 female, M. Mei leg., V. Nobile det. (VNC).

Distribution. Central South European Central Asian. The other subspecies, Hoplitis (Hoplitis) adunca ssp. contraria Tkalcu, 1974 has an Iberian Maghrebian distribution.

Remarks. New for the Aeolian Archipelago.

Hoplitis (Hoplitis) lepeletieri (Panzer, 1798)

EXAMINED MATERIAL. Vulcano, 25.IV-4.V.2009, 1 male, M. Mei leg., V. Nobile det. (VNC).

Distribution. Central South West European.

REMARKS. New for the Aeolian Archipelago.

Osmia (Helicosmia) latreillei ssp. iberoafricana Peters, 1975

EXAMINED MATERIAL. Filicudi, 14.VII.1993, 1 female, M. Mei leg., V. Nobile det. (VNC); Vulcano, 29.V-4.VI.2010, 1 male, M. Mei leg., V. Nobile det. (VNC).

Distribution. South Mediterranean Macaronesian. The nominate subspecies is South European, excluding Iberian Peninsula.

REMARKS. New for the Aeolian Archipelago.

Osmia (Pyrosmia) submicans ssp. hebraea Benoist, 1934

EXAMINED MATERIAL. Vulcano, 29.V-4.VI.2010, 1 female, M. Mei leg., V. Nobile det. (VNC).

Distribution. South Mediterranean. The nominate subspecies is Central South European.

REMARKS. New for the Aeolian Archipelago.

Rhodanthidium septemdentatum (Latreille, 1809)

EXAMINED MATERIAL. Lipari: Quattropani, 6. 
VI.1997, 1 male, G.F. Turrisi leg., V. Nobile det. (GFTC); Pianoconte, 7.VI.1997, 1 female, G.F. Turrisi leg., V. Nobile det. (GFTC).

Distribution. North Mediterranean.

Remarks. New for the Aeolian Archipelago.

\section{Stelis phaeoptera ssp. murina Perez, 1884}

EXAMINED MATERIAL. Lipari: VI.2000, 1 male, 5 females, Sinacori leg., reared from Arundo stems together with the host Osmia (Chalcosmia) sp., V. Nobile det. (VNC).

Distribution. South Mediterranean Iranian Macaronesian. The nominate subspecies is Central North European Central Asian.

Remarks. New for the Aeolian Archipelago.

Dioxys cincta (Jurine, 1807)

EXAMINED MATERIAL. Lipari: 14.IV.1968, 1 female, G. Marcuzzi leg., V. Nobile det. (DSCC).

Distribution. Central European Mediterranean African.

REMARKS. Recorded for Lipari by Nobile \& Turrisi (1996).

Megachile (Chalicodoma) parietina (Geoffroy, 1785)

EXAMINED MATERIAL. Lipari: Pianoconte, 7. VI. 1997, 1 female, G.F. Turrisi leg., V. Nobile det. (GFTC). Panarea: 23.VI.1967, 1 female, Sulfaro leg., V. Nobile det. (DSCC).

Distribution. Central South Palaearctic.

REMARKS. New for the Aeolian Archipelago.

Megachile (Chalicodoma) sicula Rossi, 1792

EXAMINED MATERIAL. Lipari: Vallone Ponte, 22. II.1967, 1 male, S. Bruno leg., V. Nobile det. (DSCC).

Distribution. South Palaearctic Macaronesian.

Remarks. New for the Aeolian Archipelago.
Megachile (Eutricharaea) apicalis Spinola, 1808

EXAMINED MATERIAL. Lipari: Pianoconte, 7. VI.1997, 1 female, G.F. Turrisi leg., V. Nobile det. (GFTC).

Distribution. Central South European Maghrebian Central Asian (extended to North America).

Remarks. Recorded for Vulcano by Comba \& Comba (2005).

Megachile (Eutricharaea) pilidens Alfken, 1923

EXAMINED MATERIAL. Vulcano: 28.VI-18. VII. 2008, 1 male, 1 female, M. Mei leg., V. Nobile det. (VNC). Vulcano: Piano, 330 m, 21.VIII.2014, 1 specimen, G. Altadonna leg., V. Nobile det. (PNC).

Distribution. Mediterranean Central European Iranian.

REMARKS. Recorded for Vulcano by Comba \& Comba (2005).

APIDAE

Xylocopa violacea (Linnaeus, 1758)

ExAmined MATERIAL. Lipari: Pianoconte, 7. VI.1997, 1 female, G.F. Turrisi leg. and det. (GFTC). Vulcano: Piano, 21.VIII.2014, 1 female, G. Altadonna leg., G.F. Turrisi det. (GAC). Vulcano: Gelso, 12.VIII.2016, 1female, G. Altadonna leg., G.F. Turrisi det. (GAC).

Distribution. Palaearctic (recorded also for Mexico).

Remarks. Recorded for Lipari, Filicudi, Panarea, and Vulcano by Pagliano \& Nobile (1993), Vicidomini (2003), and Vicidomini et al., (2005); for Stromboli by Comba \& Comba (2005). The similar $X$. valga Gerstaecker, 1872 is recorded for Stromboli by Pagliano \& Nobile (1993), Vicidomini (2003), and Vicidomini et al., (2005).

\section{Ceratina cucurbitina (Rossi, 1792)}

EXAMINED MATERIAL. Lipari: Urnazzo, 2.VI. 1997, 1 female, P. Lo Cascio leg., on Daucus sp., 
V. Nobile det. (PLCC); Chiesa Vecchia, 7.VI.1999, on cultivated Petroselinum sp. (Apiaceae), G.F. Turrisi leg., V. Nobile det. (GFTC). Vulcano: Piano, 9-22.VIII.2015, 1 male (yellow-pan trap), G. Altadonna leg., V. Nobile det. (GAC). Alicudi: Pianicello, 2.IV.1997, 1 female, Lo Cascio \& Serra leg., V. Nobile det. (PLCC).

Distribution. Central South West Palaearctic.

REMARKs. New for the Aeolian Archipelago.

Nomada fulvicornis Fabricius, 1793

EXAMINED MATERIAL. Vulcano: 25.IV-4.V.2009, 2 female, M. Mei leg., V. Nobile det. (VNC). Vulcano, 29.V-4.VI.2010, 1 male, 1 female, M. Mei leg., V. Nobile det. (VNC).
Distribution. Central South Palaearctic.

REMARKS. New to Aeolian archipelago.

Epeolus julliani Perez, 1884

EXAMINED MATERIAL. Vulcano: 3-17.VII.1993, 1 female, M. Mei leg. and det. (VNC).

Distribution. South European Anatolian.

REMARKS. New for the Aeolian Archipelago.

Pasites maculatus Jurine, 1807

EXAMINED MATERIAL. Vulcano: 3-17.VII.1993, 1 male, 2 females, M. Mei leg., V. Nobile det. (VNC).

Distribution. Central West Palaearctic.

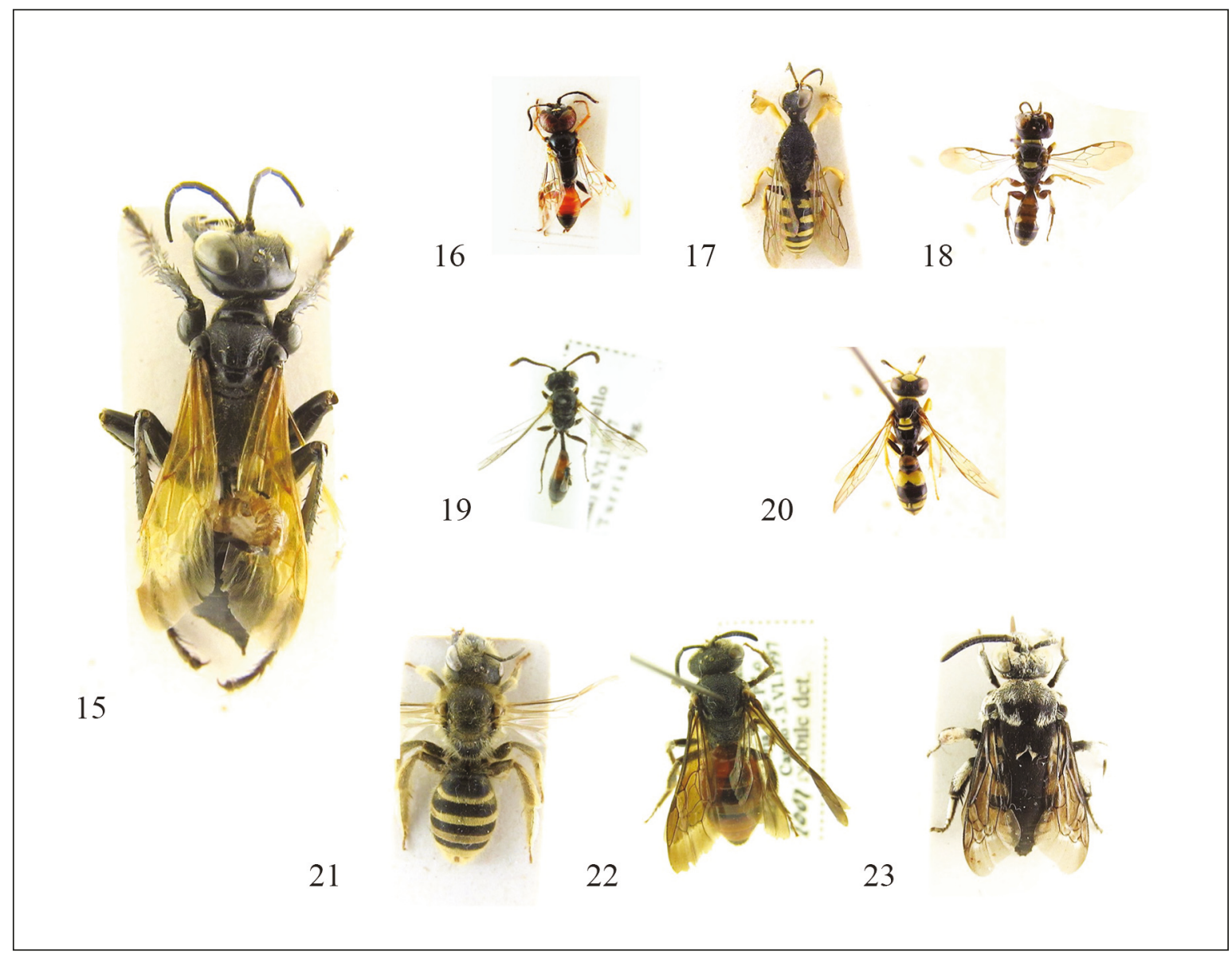

Figures 15-23. Hymenoptera of Aeolian Archipelago (not in scale). Fig. 15: Prionyx subfuscatus female (Lipari). Fig. 16: Dryudella esterinae male (Lipari). Fig. 17: Lestica clypeata male (Lipari). Fig. 18: Tracheliodes quinquenotatus female (Lipari). Fig. 19: Mimesa grandii female (Lipari). Fig. 20: Philanthus coarctatus siculus male (Lipari). Fig. 21: Halictus scabiosae female (Lipari). Fig. 22: Sphecodes albilabris female (Lipari). Fig. 23: Thyreus ramosus male (Basiluzzo). 
Remarks. New for the Aeolian Archipelago.

Eucera (Eucera) nigrescens Perez, 1879

EXAmined MATERial. Alicudi: 5.VII.1993, 1 male, 1 female, M. Mei leg., V. Nobile det. (VNC). Vulcano, 15.VII.1993, 1 female, M. Mei leg., V. Nobile det. (VNC).

Distribution. Central South European Iranian.

Remarks. New for the Aeolian Archipelago.

Tetraloniella dentata (Germar, 1839)

EXAMINED MATERIAL. Vulcano: 29.V-4.VI.2010, 1 male, M. Mei leg., V. Nobile det. (VNC).

Distribution. Central Southern Palaearctic.

Remarks. New for the Aeolian Archipelago.

Amegilla albigena (Lepelletier, 1841)

EXAMINED MATERIAL. Vulcano: 24.VII.1963, 1 female, V. Nobile leg. and det. (VNC).

Distribution. Mediterranean Central North East African.

REMARKs. New for the Aeolian Archipelago.

Amegilla garrula (Rossi, 1790)

EXAMINED MATERIAL. Lipari: Castello environs, 8.VI.1997, 1 male, G.F. Turrisi leg., V. Nobile det. (GFTC). Salina: Monte Dei Porri, 20.IX.1966, 1 female, S. Arcidiacono leg., V. Nobile det. (VNC).

DistRIBUTION. South European Iranian.

REMARKs. New for the Aeolian Archipelago.

Thyreus ramosus (Lepelletier, 1841) (Fig. 23)

EXAMINED MATERIAL. Basiluzzo: 20.VIII.1996, 1 male, P. Lo Cascio leg., V. Nobile det. (PLCC).

Distribution. South Palaearctic Macaronesian. REMARKS. New for the Aeolian Archipelago.

Anthophora (Lophanthophora) dispar Lepelletier, 1841

Examined material. Vulcano: Piano, 14.
III.2000, 2 females, 1 male, P. Lo Cascio \& S. Pasta leg., on Cytisus aeolicus Guss. ex Lindl., V. Nobile det. (PLCC).

Distribution. Mediterranean.

REMARKS. Recorded for Vulcano by Lo Cascio (2004) (sub Lophanthophora dispar) and for Alicudi by Comba \& Comba (2005).

Anthophora (Anthophora) plumipes ssp.
niger (Friese, 1896)

Examined Material. Vulcano: Piano, $330 \mathrm{~m}$ a.s.l., 2.VI.2012, 1 specimen, G. Altadonna leg., V. Nobile det. (PNC).

Distribution. Palaearctic.

Remarks. New for the Aeolian Archipelago.

Bombus (Bombus) terrestris (Linnaeus, 1758)

EXAMINED MATERIAL. Vulcano: Piano, 14.III.2000, 1 worker, P. Lo Cascio \& S. Pasta leg., on Cytisus aeolicus Guss. ex Lindl., V. Nobile det. (PLCC).

Distribution. Palaearctic.

REMARKS. Recorded for Vulcano by Lo Cascio (2004).

Apis mellifera (Linnaeus, 1758)

Examined Material. Lipari: Canale, 30.VI. 1967, 1 worker, Allegrina leg., V. Nobile det. (VNC). Lipari: Pianoconte, 7.VI.1997, 1 worker, G.F. Turrisi leg. and det. (GFTC).

Distribution. Cosmopolitan.

REMARKS. Recorded for the Aeolian Archipelago by Bouga et al. (2011), Tenore et al. (2012), Muñoz et al. (2014).

\section{DISCUSSION AND CONCLUSIONS}

Data from literature (see Introduction) recorded 98 species (including one doubtful record among Formicidae) for the archipelago; 20 of them are confirmed by the present research, and 120 are added as new records. As the whole, 218 species of Hymenoptera belonging to 24 families are currently 
known for the Aeolian Islands (Table 1). Among them, Dryudella esterinae, Nysson quadriguttatus, Miscophus aetoni, Miscophus lusitanicus, Cerceris circularis ssp. dacica and Lasioglossum (Dialictus) semilucens are recorded for the first time for Sicily, and Miscophus lusitanicus resulted also new for Italy.

Despite this remarkable increase, the hymenopteran fauna of these islands should be considered largely underestimated, especially for many groups of Terebrantia, also including those so called "micro Hymenoptera". No sawflies ("Symphyta") have yet been recorded for the Aeolian Archipelago, but it could be due to the lack of collecting efforts, as suggested by the relatively high diversity and endemism richness that characterizes this group in Sicily, where 139 species were so far recorded (Pesarini \& Turrisi, 2001; Turrisi, 2011; Liston et al., 2013); for instance, it is likely that at least some representatives of the most speciose family Tenthredinidae should be occurring also in the Aeolian Archipelago.

On the contrary, these islands harbour a relatively high diversity of Formicidae, with 49 species, and Apoidea, with 68 species belonging to 5 families, and especially Halictidae that include 29 species.

Although the available data are not sufficient to perform an exhaustive biogeographical analysis, taking into account the distributional patterns only, most of the recorded species have a wide EuropeanMediterranean distribution, and even in the Palaearctic region; moreover, the Mediterranean chorotypes seem to be well represented within the Aeolian fauna.

The Hymenoptera represent one of the four mega-diverse insect orders, and they play a fundamental role in virtually all terrestrial ecosystems as parasitoids, predators, and pollinators, being of substantial economic importance. As the faunal knowledge is likely far to be complete, data concerning population sizes and trends, distribution and conservation of Hymenoptera in the study islands are very limited and essentially based on personal observations carried out by the authors during the field-work.

Anthropogenic disturbance is not strong, but it increases during summer due to the massive occurrence of tourism. This factor, together with urban sprawl and infrastructural developments, may affect structure and dynamics of local ecosystems, as well as the fire, that recently involved large areas of the main islands (f.i., Lipari and Vulcano). The islands have also a long-term history of agricultural ex- ploitation, that was characterized by a phase of recession and general abandonment between the late 19 th and the second half of 20th century. Currently agriculture, and especially viticulture, are gradually spreading again in some islands (Lipari, Salina, Vulcano), but generally moving toward organic practices and avoiding the use of pesticides. On the other hand, the small surface and the rough morphology of the islands hinder strong agricultural changes such as mechanization.

Especially bee populations and species are sensitive to environmental changes, as observed for continental Europe where this problem, together with stress from parasites, pesticides and flower pauperization, has caused honey bee colony losses and declines of wild pollinators (Goulson et al., 2015). However, some of the study islands (Lipari, Vulcano, Filicudi and Alicudi) are now exclusively inhabited by the Sicilian subspecies of honey bee (Apis mellifera ssp. siciliana Dalla Torre, 1896), that is bred in traditional manner and not exposed to the risk of genetic pollution, as since 1990s the Municipality of Lipari has promulged an ordinance prohibiting the introduction of other bee races. In this perspective, the islands may be considered as "refuges" for threatened hymenopteran that in other areas (such as Sicily) suffer all the adverse factors above-mentioned, including the modernization of agricultural practices.

More detailed faunal knowledge, together with the extension of the researches to the local population trends and a monitoring programme, are however needed in order to recognize and prevent any negative change that could affect the Aeolian hymenopteran fauna, and consequently its role in agriculture and maintenance of plant diversity. In fact, trends in the decline of Hymenoptera, as with other invertebrates, could be underestimated because of insufficient data both on the number of species, and on the size of local populations. It is not excluded that even among invertebrates, usually considered more "resistant" to the so-called sixth mass extinction (see Leakey \& Lewin, 2015 and Kolbert, 2016), some species may disappear even before to be discovered. The development of investigations for a better understanding of the population trends, as well as improvements to the taxonomy and the ecology of the Hymenoptera, are critical for an effective conservation and management of this important faunal component, also for wider benefits associated with pollination network of the ecosystem. 


\begin{tabular}{|c|c|c|c|c|c|c|c|c|}
\hline Taxa & 통. & 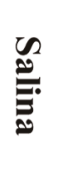 & 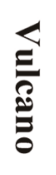 & 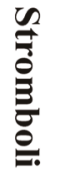 & 胥 & 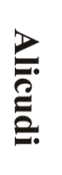 & 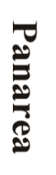 & $\frac{\sqrt{2}}{\frac{a}{2}}$ \\
\hline \multicolumn{9}{|l|}{ EVANIIDAE } \\
\hline Zeuxevania splendidula (Costa, 1884) & + & & & & & & & \\
\hline \multicolumn{9}{|l|}{ AULACIDAE } \\
\hline Pristaulacus galitae (Gribodo, 1879) & & & + & & & & & \\
\hline Pristaulacus lindae Turrisi, 2000 & & & + & & & & & \\
\hline \multicolumn{9}{|l|}{ GASTERUPTIIDAE } \\
\hline Gasteruption assectator (Linnaeus, 1758) & + & & & & & & & \\
\hline Gasteruption erythrostomum (Dahlbom, 1831) & + & & & & & & & \\
\hline Gasteruption jaculator (Linnaeus, 1758) & + & & & & & & & \\
\hline \multicolumn{9}{|l|}{ ICHNEUMONIDAE } \\
\hline Eutanyacra picta (Schrank, 1776) & & & + & & & & & \\
\hline Clistopyga incitator (Fabricius, 1793) & & & 0 & & & & & \\
\hline Zaglyptus multicolor (Gravenhorst, 1829) & & & o & & & & & \\
\hline \multicolumn{9}{|l|}{ EURYTOMIDAE } \\
\hline Eurytoma martellii Domenichini, 1960 & & 0 & & & & & & \\
\hline \multicolumn{9}{|l|}{ PTEROMALIDAE } \\
\hline Cyrtoptyx latipes (Rondani, 1874) & & 0 & & & & & & \\
\hline Scutellista cyanea Motschulsky, 1859 & & & 0 & & & & & \\
\hline \multicolumn{9}{|l|}{ EUPELMIDAE } \\
\hline Eupelmus urozonus Dalman, 1820 & & 0 & & & & & & \\
\hline \multicolumn{9}{|l|}{ EULOPHIDAE } \\
\hline Pnigalio mediterraneus Ferriére \& Delucchi, 1957 & & 0 & & & & & & \\
\hline \multicolumn{9}{|l|}{ LEUCOSPIDAE } \\
\hline Leucospis gigas Fabricius, 1793 & + & & & 0 & & & & \\
\hline \multicolumn{9}{|l|}{ CHRYSIDIDAE } \\
\hline Hedychridium cf. mediocrum Linsenmaier, 1987 & & + & & & & & & \\
\hline Hedychrum cf. micans europaeum Linsenmaier, 1959 & & + & & & & & & \\
\hline Holopyga fervida (Fabricius, 1781) & + & & + & + & & + & + & \\
\hline Pseudomalus auratus (Linnaeus, 1758) & $\bullet$ & & & & & & & \\
\hline Chrysis cf. auriceps Mader, 1936 & & & & & & & + & \\
\hline Chrysis cerastes Abeille De Perrin, 1877 & & + & & & & & & \\
\hline Chrysis ignita ignita Linnaeus, 1761 & & & 0 & & & & & \\
\hline Chrysis scutellaris Fabricius, 1794 & & & & & + & & & \\
\hline Pseudochrysis neglecta (Shuckard, 1837) & & & + & & & & & \\
\hline \multicolumn{9}{|l|}{ DRYINIDAE } \\
\hline Gonatopus lunatus Klug, 1810 & & & 0 & & & & & \\
\hline \multicolumn{9}{|l|}{ TIPHIIDAE } \\
\hline Tiphia lepeletieri Berland, 1925 & + & & & & & & & \\
\hline Meria tripunctata (Rossi, 1790) & $\bullet$ & & + & & & & & \\
\hline Meria dorsalis (Fabricius, 1804) & $\bullet$ & & + & & & & & \\
\hline
\end{tabular}




\begin{tabular}{|c|c|c|c|c|c|c|c|}
\hline \multicolumn{8}{|l|}{ SCOLIIDAE } \\
\hline Megascolia bidens (Linnaeus, 1767) & 0 & & $\bullet$ & & & & \\
\hline Megascolia maculata (Drury, 1773) & $\bullet$ & 0 & $\bullet$ & & & & \\
\hline Scolia hirta (Schrank, 1781) & + & & & & & & \\
\hline Scolia carbonaria (Linnaeus, 1767) & + & & & & & & \\
\hline Scolia sexmaculata (Müller, 1766) & 0 & & 0 & & & & \\
\hline Colpa sexmaculata (Fabricius, 1781) & o & & + & 0 & & & \\
\hline Dasyscolia ciliata (Fabricius, 1787) & & & + & & & & \\
\hline \multicolumn{8}{|l|}{ MUTILLIDAE } \\
\hline Bidecoloratilla leopoldina (Invrea, 1955) & o & o & O & & & & \\
\hline Myrmilla calva (Villiers, 1789) & 0 & & 0 & & & & \\
\hline Ronisia brutia brutia (Petagna, 1787) & 0 & 0 & 0 & & 0 & 0 & \\
\hline Ronisia ghilianii (Spinola, 1843) & 0 & & & & & 0 & \\
\hline Tropidotilla litoralis (Petagna, 1787) & 0 & & 0 & & & & \\
\hline Nemka viduata viduata (Pallas, 1773) & 0 & & 0 & 0 & & & \\
\hline Physetopoda pusilla (Klug, 1835) & 0 & & & & & & \\
\hline Smicromyrme ausonia Invrea, 1950 & 0 & & & & & & \\
\hline Smicromyrme partita (Klug, 1835) & 0 & & & & & & \\
\hline Smicromyrme ruficollis ruficollis (Fabricius, 1793) & 0 & & 0 & o & & & \\
\hline Smicromyrme sulcisia Invrea, 1955 & 0 & & 0 & & & & \\
\hline Smicromyrme trinotata (Costa, 1858) & 0 & & & & & & \\
\hline Dasylabris maura maura (Linnaeus, 1758) & O & & & & & & \\
\hline \multicolumn{8}{|l|}{ POMPILIDAE } \\
\hline Pompilus cinereus Fabricius, 1798 & & & + & & & & \\
\hline Agenioideus ciliatus (Lepelletier, 1845) & + & & & & & & \\
\hline Anoplius viaticus (Linnaeus, 1758) & + & & & & & & \\
\hline Aporus bicolor Spinola, 1808 & + & & & & & & \\
\hline Auplopus carbonarius (Scopoli, 1763) & + & & & & & & \\
\hline Priocnemis (Priocnemis) propinqua (Lepelletier, 1845) & + & & & & & & \\
\hline \multicolumn{8}{|l|}{ VESPIDAE EUMENINAE } \\
\hline Leptochilus (Neoleptochilus) regulus (Saussure, 1855) & & & 0 & & & & \\
\hline Stenodynerus fastidiosissimus s. str. (Saussure, 1855) & & & O & & & & \\
\hline Ancistrocerus auctus auctus (Fabricius, 1793) & O & & & & & & \\
\hline Ancistrocerus gazella (Panzer, 1798) & & & O & & & & \\
\hline Ancistrocerus longispinosus longispinosus (Saussure, 1855) & O & O & $\bullet$ & & & & \\
\hline Eumenes coarctatus maroccanus Gusenleitner, 1972 & & & 0 & & & & \\
\hline Eumenes mediterraneus mediterraneus Kriechbaumer, 1879 & O & & + & & & & \\
\hline Delta unguiculatum (Villers, 1789) & + & & & & & & \\
\hline Rynchium oculatum (Fabricius, 1781) & & & $\bullet$ & & & & \\
\hline \multicolumn{8}{|l|}{ VESPIDAE VESPINAE } \\
\hline Vespa crabro crabro Linnaeus, 1758 & & & + & & & & \\
\hline Polistes (Polistes) dominula (Christ, 1791) & & & + & & & & \\
\hline Polistes (Polistes) gallicus (Linnaeus, 1767) & & & + & & & & \\
\hline Polistes (Polistes) nimpha (Christ, 1791) & & & + & & & & \\
\hline
\end{tabular}




\begin{tabular}{|c|c|c|c|c|c|c|c|c|}
\hline Vespula (Paravespula) germanica (Fabricius, 1793) & + & & + & & & & & \\
\hline \multicolumn{9}{|l|}{ SPHECIDAE } \\
\hline Sceliphron destillatorium (Illiger, 1807) & & & & & & & 0 & \\
\hline Sceliphron spirifex (Linnaeus, 1758) & & & o & & & & & \\
\hline Ammophila heydeni Dahlbom, 1845 & + & & & & & & & \\
\hline Prionyx subfuscatus (Dahlbom, 1845) & + & & & & & & & \\
\hline Prionyx lividocinctus (A. Costa, 1858) & + & & & & & & & \\
\hline Sphex funerarius Gussakovskij, 1934 & + & & & & & & & \\
\hline \multicolumn{9}{|l|}{ CRABRONIDAE } \\
\hline Dryudella esterinae Pagliano, 2001 & + & & & & & & & \\
\hline Dryudella tricolor (Van der Linden, 1829) & + & & & & & & & \\
\hline Harpactus laevis (Latreille, 1792) & + & & & & & & & \\
\hline Nysson quadriguttatus Spinola, 1808 & & & + & & & & & \\
\hline Crossocerus distinguendus (Morawitz, 1866) & + & & & & & & & \\
\hline Crossocerus quadrimaculatus (Fabricius, 1793) & + & & & & & & & \\
\hline Lestica clypeata (Schreber, 1759) & + & & & & & & & \\
\hline Tracheliodes quinquenotatus (Jurine,1807) & + & & + & & & & & \\
\hline Tachysphex costae (De Stefani-Perez, 1882) & & & + & & & & & \\
\hline Tachysphex nitidior Beaumont, 1940 & & & + & & & & & \\
\hline Miscophus eatoni Saunders, 1903 & & & + & & & & & \\
\hline Miscophus lusitanicus Andrade, 1952 & & & & & & & + & \\
\hline Miscophus pretiosus Kohl, 1884 & & & + & & & & & \\
\hline Solierella compedita (Piccioli, 1869) & + & & & & & & + & \\
\hline Pison atrum (Spinola, 1808) & + & & & & & & & \\
\hline Trypoxylon kolazyi Kohl, 1893 & + & & + & & & & & \\
\hline Trypoxylon deceptorium Antropov, 1991 & & & + & & & & & \\
\hline Trypoxylon scutatum Chevrier, 1867 & + & & + & & & & & \\
\hline Ammoplanus perrisi Giraud, 1869 & + & & & & & & & \\
\hline Ammoplamus (Ammoplanellus) simplex Gussakovskij, 1952 & & & 0 & & & & & \\
\hline Passaloecus pictus Ribaut, 1952 & + & & & & & & & \\
\hline Pemphredon austriaca (Kohl, 1888) & + & & & & & & & \\
\hline Mimesa grandii Maidl, 1933 & + & & & & & & & \\
\hline Cerceris circularis dacica Schletterer, 1887 & + & & & & & & & \\
\hline Philanthus coarctatus siculus Giordani Soika, 1944 & + & & & & & & & \\
\hline \multicolumn{9}{|l|}{ FORMICIDAE } \\
\hline Aphaenogaster pallida (Nylander, 1848) & 0 & o & & & + & & & $2+$ \\
\hline Aphaenogaster semipolita (Nylander, 1856) & 0 & 0 & & & 0 & & 0 & \\
\hline Aphaenogaster splendida (Roger, 1859) & & & & 0 & & & & \\
\hline Aphaenogaster subterranea (Latreille, 1798) & 0 & o & & 0 & 0 & O & o & $1+$ \\
\hline Camponotus aethiops (Latreille, 1798) & 0 & & & & & & & \\
\hline Camponotus lateralis (Olivier, 1791) & 0 & 0 & $\bullet$ & 0 & 0 & & 0 & \\
\hline Camponotus micans (Nylander, 1856) & $\bullet$ & 0 & 0 & & 0 & & & \\
\hline Camponotus nylanderi Emery, 1921 & - & 0 & 0 & 0 & • & + & 0 & $\begin{array}{l}1 \bullet \\
4+\end{array}$ \\
\hline Camponotus piceus (Leach, 1825) & 0 & & & & & & & \\
\hline
\end{tabular}




\begin{tabular}{|c|c|c|c|c|c|c|c|c|}
\hline Camponotus ruber Emery, 1925 & 0 & & & & & & 0 & \\
\hline Crematogaster scutellaris (Olivier, 1791) & - & 0 & 0 & o & 0 & & o & \\
\hline Crematogaster sordidula (Nylander, 1849) & & 0 & & & & & 0 & \\
\hline Formica cunicularia Latreille, 1798 & 0 & & & & & & & \\
\hline Lepisiota frauenfeldi (Mayr, 1855) & o & & & & & & & \\
\hline Lepisiota nigra (Dalla Torre, 1893) & o & 0 & o & & & & $\mathrm{O}$ & $1+$ \\
\hline Messor barbarus (Linnaeus, 1767) & & 0 & & & & & & \\
\hline Messor bouvieri Bondroit, 1918 & 0 & 0 & 0 & 0 & 0 & & 0 & \\
\hline Messor capitatus (Latreille, 1798) & - & & & & & & & \\
\hline Messor ibericus Santschi, 1931 & & 0 & & & o & & & \\
\hline Messor meridionalis (André, 1882) & 0 & & & & & & & \\
\hline Messor minor ssp. calabricus Santschi, 1927 & & & & & & & & $2 \bullet$ \\
\hline Messor structor (Latreille, 1798) & $\bullet$ & & & & & & & \\
\hline Nylanderia sp. & & & & o & & & & \\
\hline Oxyopomyrmex saulcyi Emery, 1889 & 0 & & & & & & & \\
\hline Pheidole pallidula (Nylander, 1848) & 0 & 0 & 0 & • & 0 & & o & \\
\hline Plagiolepis pallescens Lomnicki, 1925 & o & 0 & o & O & & O & O & $1+$ \\
\hline Plagiolepis pygmaea (Latreille, 1798) & o & & & & 0 & & & \\
\hline Ponera coarctata (Latreille, 1802) & 0 & 0 & & & & $\bullet$ & & \\
\hline Tetramorium bicarinatum (Nylander, 1846) & 0 & & & & & & 0 & \\
\hline Tetramorium diomedeum Emery, 1908 & & & & & o & & & \\
\hline Tetramorium immigrans Santschi, 1927 & & & & & & & o & \\
\hline Tetramorium punctatum Santschi, 1927 & 0 & 0 & 0 & 0 & 0 & & 0 & $4+$ \\
\hline Tetramorium semilaeve André, 1883 & 0 & 0 & 0 & 0 & 0 & 0 & 0 & \\
\hline Lasius myops Forel, 1894 & o & O & o & & & & & \\
\hline Lasius casevitzi Seifert \& Galkowski, 2016 & o & 0 & & & & 0 & & \\
\hline Lasius emarginatus (Olivier, 1792) & 0 & 0 & & & & & & \\
\hline Lasius lasioides (Emery, 1869) & 0 & 0 & 0 & 0 & 0 & 0 & 0 & \\
\hline Linepithema humile (Mayr, 1868) & & & & 0 & 0 & & & \\
\hline Monomorium subopacum (F. Smith, 1858) & 0 & & & O & 0 & & O & \\
\hline Solenopsis fugax (Latreille, 1798) & 0 & & & & & & & \\
\hline Solenopsis latro Forel, 1894 & 0 & 0 & & 0 & 0 & & & \\
\hline Strongylognathus destefanii Emery, 1915 & & & 0 & & & & & \\
\hline Strumigenys membranifera Emery, 1869 & & & & O & & & & \\
\hline Tapinoma erraticum (Latreille, 1798) & 0 & 0 & 0 & & & & & \\
\hline Tapinoma nigerrimum complex & 0 & 0 & 0 & 0 & 0 & & 0 & \\
\hline Temnothorax exilis (Emery, 1869) & 0 & 0 & 0 & 0 & 0 & 0 & o & \\
\hline Temnothorax lichtensteini (Bondroit, 1918) & & O & & & & & & \\
\hline Temnothorax mediterraneus Ward, Brady, Fisher \& Schultz, 2014 & 0 & & 0 & 0 & 0 & & 0 & \\
\hline Wasmannia auropunctata (Roger, 1863) & & & & & & & & $2 ?$ \\
\hline \multicolumn{9}{|l|}{ COLLETIDAE } \\
\hline Colletes nigricans Gistel, 1857 & & + & & & & & & \\
\hline Hylaeus (Paraprosopis) clypearis (Schenck, 1853) & & & & & & & + & \\
\hline Hylaeus (Dentigera) imparilis Förster, 1871 & + & & & & & & & \\
\hline Hylaeus (Spatulariella) punctatus (Brullé, 1832) & & + & & & & & & \\
\hline
\end{tabular}




\begin{tabular}{|c|c|c|c|c|c|}
\hline \multicolumn{6}{|l|}{ ANDRENIDAE } \\
\hline Andrena (Charitandrena) hattorfiana dimidiata Brullé, 1832 & & + & & & \\
\hline Andrena (Euandrena) ruficrus Nylander, 1848 & + & & & & \\
\hline Andrena (Melandrena) morio morio Brullé, 1832 & & + & & + & \\
\hline Andrena (Melandrena) nigroaenea s.str. (Kirby, 1802) & & & + & & \\
\hline Andrena (Melandrena) nitida nitida (Müller, 1776) & & + & & & \\
\hline Andrena (Plastandrena) pilipes Fabricius, 1781 & & + & & & \\
\hline Andrena (Scitandrena) scita Eversmann, 1852 & & + & & & \\
\hline Andrena (Taeniandrena) wilkella (Kirby, 1802) & & + & & & \\
\hline \multicolumn{6}{|l|}{ HALICTIDAE } \\
\hline Pseudapis bispinosa (Brullé, 1832) & & + & & & \\
\hline Ceylalictus variegatus (Olivier, 1789) & & + & & & \\
\hline Nomioides facilis (Smith, 1853) & + & & & & \\
\hline Nomioides minutissimus (Rossi, 1790) & & + & & & \\
\hline Halictus (Halictus) quadricinctus (Fabricius, 1776) & & + & & + & \\
\hline Halictus (Hexataenites) fulvipes (Klug, 1817) & + & + & & + & \\
\hline Halictus (Hexataenites) scabiosae (Rossi, 1790) & + & & & & \\
\hline Halictus (Monilapis) compressus Walckenaer, 1802 & + & & & & \\
\hline Halictus (Seladonia) gemmeus Dours, 1872 & & + & & & \\
\hline Halictus (Seladonia) smaragdulus Vachal, 1895 & & + & & & \\
\hline Halictus (Tytthalictus) asperulus Perez, 1895 & & + & & + & \\
\hline Halictus (Tytthalictus) maculatus Smith, 1848 & & & & + & \\
\hline Lasioglossum (Dialictus) nitidulum (Fabricius, 1804) & + & & & & \\
\hline Lasioglossum (Dialictus) planulum (Perez, 1903) & + & & & & \\
\hline Lasioglossum (Dialictus) semilucens (Alfken, 1914) & + & & & & \\
\hline Lasioglossum (Sphecodogastra) immunitum sillatum (Warncke, 1981) & & + & & & \\
\hline Lasioglossum (Sphecodogastra) malachurum (Kirby, 1802) & + & & & & \\
\hline Lasioglossum (Sphecodogastra) opacum Perez, 1895 & + & + & & & \\
\hline Lasioglossum (Sphecodogastra) nigripes (Lepelletier, 1841) & + & & & & \\
\hline Lasioglossum (Sphecodogastra) pauxillum (Schenck, 1853) & & & & + & \\
\hline Lasioglossum (Sphecodogastra) subhirtum (Lepelletier, 1841) & + & & & & \\
\hline Lasioglossum (Lasioglossum) albocinctum (Lucas, 1849) & & + & & & \\
\hline Lasioglossum (Lasioglossum) bimaculatum (Dours, 1872) & + & + & & & \\
\hline Lasioglossum (Lasioglossum) costulatum (Kriechbaumer, 1873) & + & & & & \\
\hline Lasioglossum (Lasioglossum) leucozonium cedri Ebmer, 1976 & + & & & & \\
\hline Sphecodes albilabris (Fabricius, 1793) & + & & & & \\
\hline Sphecodes gibbus (Linnaeus, 1758) & & + & & & \\
\hline Sphecodes monilicornis quadratus Meyer, 1919 & & + & & & \\
\hline Sphecodes spinulosus Hagens, 1875 & & + & & & \\
\hline \multicolumn{6}{|l|}{ MEGACHILIDAE } \\
\hline Heriades (Heriades) truncorum (Linnaeus, 1758) & & + & & & \\
\hline Hoplitis (Hoplitis) adunca adunca (Panzer, 1798) & & + & & & \\
\hline Hoplitis (Hoplitis) lepeletieri (Panzer, 1798) & & + & & & \\
\hline Osmia (Helicosmia) latreillei iberoafricana Peters, 1975 & & + & + & & \\
\hline Osmia (Pyrosmia) submicans hebraea Benoist, 1934 & & + & & & \\
\hline
\end{tabular}




\begin{tabular}{|c|c|c|c|c|c|c|c|c|}
\hline Rhodanthidium septemdentatum (Latreille, 1809) & + & & & & & & & \\
\hline Stelis phaeoptera murina Perez, 1884 & + & & & & & & & \\
\hline Dioxys cincta (Jurine, 1807) & + & & & & & & & \\
\hline Megachile (Chlicodoma) parietina (Geoffroy, 1785) & + & & & & & & + & \\
\hline Megachile (Chalicodoma) sicula Rossi, 1792 & + & & & & & & & \\
\hline Megachile (Eutricharaea) apicalis Spinola, 1808 & + & & O & & & & & \\
\hline Megachile (Eutricharaea) pilidens Alfken, 1924 & & & - & & & & & \\
\hline Anthidium manicatum (Linnaeus, 1758) & & & o & & & & & \\
\hline Anthidium florentinum (Fabricius, 1775) ${ }^{1}$ & & & & 0 & & & & \\
\hline \multicolumn{9}{|l|}{ APIDAE } \\
\hline Xylocopa (Xylocopa) valga Gerstaecker 1872 & & & & 0 & & & & \\
\hline Xylocopa (Xylocopa) violacea (Linnaeus, 1758) & $\bullet$ & & $\bullet$ & 0 & 0 & & 0 & \\
\hline Ceratina cucurbitina (Rossi, 1792) & + & & + & & & + & & \\
\hline Nomada fulvicornis Fabricius, 1793 & & & + & & & & & \\
\hline Epeolus julliani Perez, 1884 & & & + & & & & & \\
\hline Pasites maculatus Jurine, 1807 & & & + & & & & & \\
\hline Eucera (Eucera) nigrescens Perez, 1879 & & & + & & & + & & \\
\hline Tetraloniella dentata (Germar, 1839) & & & + & & & & & \\
\hline Amegilla albigena (Lepelletier, 1841) & & & + & & & & & \\
\hline Amegilla garrula (Rossi, 1790) & + & + & & & & & & \\
\hline Thyreus ramosus (Lepelletier, 1841) & & & & & & & & $3+$ \\
\hline Anthophora (Lophanthophora) dispar Lepelletier, 1841 & & & 0 & & & 0 & & \\
\hline Anthophora (Anthophora) plumipes niger (Friese, 1896) & & & + & & & & & \\
\hline Bombus (Bombus) terrestris (Linnaeus, 1758) & & & $\bullet$ & & & & & \\
\hline Apis mellifera (Linnaeus, 1758) & $\bullet$ & & & & & & & \\
\hline
\end{tabular}

Table 1. Summary of Hymenoptera from Aeolian archipelago. Legend: $\odot=$ bibliographic record only; $\bullet=$ record confirmed by the present study; += new record; ? = doubtful record. Islets: 1 = Strombolicchio; 2 = Lisca Bianca; $3=$ Basiluzzo; $4=$ Scoglio Faraglione. 1 observed and photographed in Stromboli as reported on 06.07.2011, https://www.naturamediterraneo. com/forum/topic.asp?TOPIC_ID=146201.

\section{ACKNOWLEDGEMENTS}

We are grateful to Maurizio Mei (Rome, Italy), Pietro Niolu (Sassari, Italy), Marcello Romano (Capaci, Palermo, Italy), and Carlo Violani (Milan, Italy) for allowing us the study of their material; Paolo Rosa (Milan, Italy) for checking and identifying Chrysididae, Fabrizio Rigato (Milan, Italy) for identifying Formicidae.

\section{REFERENCES}

Baccetti B., 1967. Notulae Orthopterologicae. XXII. Il genere Myrmecophilus Berth. in Italia. Redia, 50: 133.
Baroni Urbani C., 1964. Studi sulla mirmecofauna d'Italia. II. Formiche di Sicilia. Atti dell'Accademia gioenia di Scienze naturali, (6) 16: 25-66.

Baroni Urbani C., 1971. Catalogo delle specie di Formicidae in Italia. Memorie della Società entomologica italiana, 50: 1-289. http://doi.org/10.5281/zenodo. 26781

Boni Bartalucci M., 2012. European Myzininae (Hymenoptera: Tiphiidae). Onychium, 9: 121-191.

Boni Bartalucci M., 2013. Fauna Europaea: Tiphiidae. In: Mitroiu M.D., Fauna Europaea: Hymenoptera. Fauna Europaea version 2017.06, https://fauna-eu.org (accessed March 22, 2020).

Borsato W. \& Turrisi G.F., 2004. Contributo alla conoscenza degli Eumenidae di Sicilia (Hymenoptera, Vespoidea). Bollettino del Museo civico di Storia naturale di Venezia, 55: 127-150. 
Borowiec L., Galkowski C. \& Salata S., 2015. What is Tetramorium semilaeve André, 1883? (Hymenoptera, Formicidae). ZooKeys, (512): 39-62. https://doi.org/ 10.3897/zookeys.512.10006

Bouga M., Alaux C., Bienkowska M., Büchler R., Carreck N.L., Cauia E., Chlebo R., Dahle B., Dall'Olio R., De La Rúa P., Gregorc A., Ivanova E., Kence A., Kence M., Kezic N., Kiprijanovska H., Kozmus P., Kryger P., Le Conte Y., Lodesani M., Murilhas A.M., Siceanu A., Soland G., Uzunov A. \& Wilde J., 2011. A review of methods for discrimination of honey bee populations as applied to European beekeeping. Journal of apicultural Research, 50: 51-84. https:// doi.org/10.3896/IBRA.1.50.1.06

Carbonell-Font R. \& Turrisi G.F., 2017. First record of Pristaulacus lindae Turrisi, 2000 from Iberian Peninsula (Hymenoptera, Aulacidae). Boletin de la Asociacíon española de Entomologia, 41: 285-291.

Ceccolini F. \& Barbagli F., 2014. Nuovi dati corologici per gli Imenotteri delle isole minori dell'Italia meridionale (Hymenoptera Scoliidae, Sphecidae, Apidae). Il Naturalista siciliano, 38: 93-111.

Comba L. \& Comba M., 2005. Insecta Hymenoptera Aculeata Apoidea (partim). In: Ruffo S. \& Stoch F. (eds.), Checklist e distribuzione della Fauna italiana. Memorie del Museo civico di Storia naturale di Verona (Scienze della Vita), 2: 275-277.

Costantino G., 1937. Contributo alla conoscenza delle Cocciniglie delle Isole Eolie. Annali della reale Stazione sperimentale di Agrumicultura e Frutticultura di Acireale, 15: 235-242.

Czösz S. \& Schulz A., 2010. A taxonomic review of the Palaearctic Tetramorium ferox species-complex (Hymenoptera, Formicidae). Zootaxa, 2401: 1-29. http:// dx.doi.org/10.11646/zootaxa.2401.1.1

Daane K.M. \& Johnson M.W., 2010. Olive fruit fly: managing an ancient pest in modern times. Annual Review of Entomology, 55: 151-169. http://dx.doi.org /10.1146/annurev.ento.54.110807.090553

Dall’Olio R., Muñoz I., De La Rúa P. \& Lodesani M., 2008. Estimating introgression in Apis mellifera sicula populations: are the conservation island really effective? pp. 23-24 in: Teal J., Osborne J.L. \& Paxton R.J. (Eds.), EurBee3, Proceedings of the 3rd European Conference on Apidology (Belfast, 8-11 September 2008).

Ebmer A.W., 1995. Asiatische Halictidae, 3. Die Artengruppe der Lasioglossum carinate-Evylaeus (Insecta: Hymenoptera: Apoidea: Halictidae: Halictinae). Linzer biologische Beiträge, 27: 525-652.

Focarile A., 1972. Ricerche entomologiche nell'arcipelago delle Eolie e nell'isola di Ustica (Sicilia). II. La coleotterofauna dello stagno salmastro di Punta Lingua nell'Isola di Salina. Memorie della Società entomologica italiana, 51: 19-37.

Gayubo F.S., Borsato W. \& Osella G., 1991. Esfecidos italianos de la coleccion del Museo civico de Historia natural de Verona (Italia) (Hymenoptera: Sphecidae). Bollettino del Museo civico di Storia naturale di Verona, 15 [1988]: 383-424.

Genduso P. \& Mineo A., 1974. Ricerche di ecologia del Dacus oleae Gmel. I. Prime osservazioni sull'infestazione dacica nell'isola di Vulcano. Bollettino dell'Istituto di Entomologia agraria e dell'Osservatorio fitopatologico di Palermo, 8: 77-111.

Generani M., Pagliano G. \& Scaramozzino P.L., 2005. Insecta Hymenoptera Scolioidea (Tiphiidae esclusi). In: Ruffo S. \& Stoch F. (Eds.), Checklist e distribuzione della fauna italiana. Memorie del Museo civico di Storia naturale di Verona (Scienze della Vita), 2: 273-274.

Goulson D., Nicholls E., Botías C. \& Rotheray E.L., 2015. Bee declines driven by combined stress from parasites, pesticides, and lack of flowers. Science: 347.10.1126/science. 1255957

Jucker C., Rigato F. \& Regalin R., 2008. Exotic ant records from Italy (Hymenoptera, Formicidae). Bollettino di Zoologia agraria e Bachicoltura, (2) 40: 99-107.

Kolbert E., 2016. La sesta estinzione. Una storia innaturale. BEAT, Milano.

Korenko S., 2017. Ecology of spider parasitoids: koinobiont ectoparasitoids from Polysphincta genus group (Ichneumonidae, Ephialtini). Habilitation thesis in Ecology, Palacký University Olomouc, Faculty of Science.

Korenko S., Di Giovanni F., 2019. Spider parasitoids of tribe Ephialtini (Hymenoptera, Ichneumonidae, Pimplinae) in Italy and their host association. Acta Zoologica Bulgarica, 71: 473-486.

Kutter H., 1927. Ein myrmekologischer Streifzug durch Sizilien. Folia myrmecologica et termitologica, 1: 94-104; (8-9): 135-136. http://doi.org/10.5281/zenodo. 26144

Leakey R. \& Lewin R., 2015. La sesta estinzione. La vita sulla Terra e il futuro del genere umano. Bollati Boringhieri, Torino.

Liston A.D., Jacobs H.J. \& Turrisi G.F., 2013. New data on the sawfly fauna of Sicily (Hymenoptera: Xyeloidea, Tenthredinoidea, Pamphilioidea, Cephoidea, Orussoidea). Entomologist's Monthly Magazine, 149: 29-65.

Lo Cascio P., 2004. Preliminary observations on the insect fauna associated with two threatened plant species, Bassia saxicola (Guss.) A.J. Scott and Cytisus aeolicus Guss., on the Aeolian Islands (Southern Tyrrhenian Sea). Il Naturalista siciliano, 28: 1155-1169.

Lo Cascio P., 2015. Worldwide checklist of the island mutillid wasps (Hymenoptera Mutillidae). Biodiversity Journal, 6: 529-592.

Lo Cascio P., 2017. Luoghi e Natura di Sicilia 1. Le Isole Eolie. Danaus, Palermo, 316 pp. 
Lo Cascio P. \& Navarra E., 2003. Guida naturalistica alle Isole Eolie. La vita in un arcipelago vulcanico. L'Epos, Palermo, 265 pp.

Lo Cascio P. \& Romano M., 2004. Mutillid wasps of the Italian minor islands (Hymenoptera Mutillidae). Bollettino della Società entomologica italiana, 136: 227250.

Lo Cascio P., Sforzi A. \& Boni Bartalucci M., 1998. I Mutillidi del Parco Naturale della Maremma (Hymenoptera Aculeata: Mutillidae). Frustula entomologica, 20 [1997]: 127-135.

Lo Cascio P., Cecchi B., Abbazzi P. \& Arnone M., 2006. A contribution to the knowledge of the Coleoptera of the Aeolian Archipelago (S Tyrrhenian) (Insecta, Coleoptera). Il Naturalista siciliano, 30: 91-116.

Matteini Palmerini M., 1992. Mutillidae del Museo civico di Storia naturale di Verona (Hymenoptera). Bollettino del Museo civico di Storia naturale di Verona, 16 [1989]: 187-226.

Mei M., 1998. Lasius (Cautolasius) myrmidon n. sp.: a new hypogaeic ant from Greece (Hymenoptera Formicidae). Bollettino della Società entomologica italiana, 130: 177-182.

Mei M., 2008. Note faunistiche su alcuni Crabronidi della fauna italiana (Hymenoptera, Crabronidae). Bollettino dell'Associazione romana di Entomologia, 63: 173-182.

Monastero S., 1965. La lutte biologique "artificielle" contre le Dacus oleae Gmel. au moyen des Opius concolor Szepl. siculus Mon. Bollettino dell'Istituto di Entomologia agraria e dell'Osservatorio fitopatologico di Palermo, 6: 7-16.

Monastero S., Delanoue P., 1965. Lotta biologica artificiale contro la Mosca delle olive (Dacus oleae Gmel.) a mezzo dell'Opius concolor Sz. siculus Mon. nelle Isole Eolie (Sicilia). Bollettino dell'Istituto di Entomologia agraria e dell'Osservatorio fitopatologico di Palermo, 6: 61-97.

Monastero S., Delanoue P., 1966a. Un grande esperimento di lotta biologica artificiale contro la Mosca delle olive (Dacus oleae G.) a mezzo dell'Opius c. Sz. siculus Mon. in Sicilia (Palermo, Luglio-Ottobre 1966). Bollettino dell'Istituto di Entomologia agraria e dell'Osservatorio fitopatologico di Palermo, 6: 145-196.

Monastero S., Delanoue P., 1966b. Lutte biologique experimentale contre la mouche de l'olive (Dacus oleae Gmel.) au moyen d'Opius concolor Szepl. siculus Mon. dans les Îles Eoliennes (Sicile) en 1965. Entomophaga, 11: 144-432.

Monastero S. \& Genduso P., 1964a. Prova di "lotta biologica artificiale" contro la mosca delle olive (Dacus oleae Gmel.) eseguita nell'isola di Salina (Eolie) nel 1963. Bollettino dell'Istituto di Entomologia agraria e dell'Osservatorio fitopatologico di Palermo, 5: 143-153.
Monastero S., Genduso P., 1964b. Esperimenti di lotta biologica artificiale contro la Mosca delle olive (Dacus oleae Gmel.) eseguiti nel 1964 nell'isola di Salina (Eolie). Bollettino dell'Istituto di Entomologia agraria e dell'Osservatorio fitopatologico di Palermo, 5: 281-289.

Muñoz I., Dall'Olio R., Lodesani M., De La Rûa P., 2014. Estimating introgression in Apis mellifera siciliana populations: are the conservation islands really effective? Insect Conservation \& Diversity, 7: 563-571. https://doi.org/10.1111/icad.12092

Nobile V., Turrisi G.F., 1996. Contributo alla conoscenza degli Apoidei di Sicilia. XIII. Le tribù Lithurgini, Dioxyni, Stelidini (Insecta, Hymenoptera, Megachilidae). Bollettino dell'Accademia gioenia di Scienze naturali, 29: 15-26.

Noyes J.S. 2019. Universal Chalcidoidea Database. World Wide Web electronic publication. http: //www.nhm.ac.uk/chalcidoids [accessed 13.04.2020]

Olmi M., 1999. Fauna d'Italia. XXXVII. Hymenoptera Dryinidae - Embolemidae. Calderini, Bologna, 425 pp.

Olmi M., 2005. Insecta Hymenoptera Drynidae Embolemidae Sclerogibbidae. In: Ruffo S. \& Stoch F. (Eds.), Checklist e distribuzione della Fauna italiana. Memorie del Museo civico di Storia naturale di Verona (Scienze della Vita), 2: 271-272.

Pagliano G., 1987. Methochidae e Scoliidae italiani (Hymenoptera). Bollettino del Museo civico di Storia naturale di Venezia, 37 [1986]: 157-181.

Pagliano G., 2003. Ricerche imenotterologiche nelle Isole di Lampedusa e Pantelleria (Hymenoptera Apocrita). Il Naturalista siciliano, 27: 115-149.

Pagliano G. \& Nobile V., 1993. Il genere Xylocopa Latreille, 1802 in Italia (Hymenoptera, Apoidea). Bollettino dell'Accademia Gioenia di Scienze naturali, 26 (342): 133-144.

Pagliano G. \& Scaramozzino P.L., 1995. Hymenoptera Gasteruptionidae, Ichneumonidae e Aculeata (esclusi Chrysidoidea, Mutillidae e Formicidae). In: Massa B. (ed.), Arthropoda di Lampedusa, Linosa e Pantelleria (Canale di Sicilia, Mar Mediterraneo). Il Naturalista siciliano, 19 (suppl.): 723-738.

Pavesi M. \& Rosa P., 2013. La collezione di Crisidi (Hymenoptera, Chrysididae) del Museo Civico di Storia Naturale di Verona. Bollettino del Museo civico di Storia naturale di Verona, 37: 47-66.

Pesarini F. \& Turrisi G.F., 2001. Contributo alla conoscenza dei Sinfiti di Sicilia (Hymenoptera Symphyta). Memorie della Società entomologica italiana, 80: 183-221.

Queiroz A.I. \& Alves D., 2016. People, transports and the spread of the Argentine ant in Europe, from c. 1850 to present. Cultura, Espaço \& Memoria, 7: 37 61. 
Riggio G. \& De Stefani Perez T., 1888. Sopra alcuni Imenotteri dell'Isola di Ustica. Nota. Il Naturalista siciliano, 7: 145-150.

Ruffo S. \& Stoch F. (Eds.), 2005. Checklist e distribuzione della fauna italiana. Memorie del Museo civico di Storia naturale di Verona, 2 serie, Sezione Scienze della Vita 16.

Sanetra M., Güsten R. \& Schulz A., 1999. On the taxonomy and distribution of Italian Tetramorium species and their social parasites (Hymenoptera Formicidae). Memorie della Società entomologica italiana, 77: 317-357.

Santschi F., 1927. A propòs du Tetramorium caespitum L. Folia myrmecologica et termitologica, 1: 52-58.

Santschi F., 1934. Fourmis d'une Croisière. Bulletin et Annales de la Société entomologique de Belgique, 74: 275-282. https://doi.org/10.5281/zenodo.14375

Schär S., Menchetti M., Schifani E., Hinojosa J.C., Platania L., Dapporto L. \& Vila R., 2020. Integrative biodiversity inventory of ants from a Sicilian archipelago reveals high diversity on young volcanic islands (Hymenoptera: Formicidae). Organisms Diversity \& Evolution, https://doi.org/10.1007/s13127020-00442-3.

Schmid-Egger C., 2003. New records of "Sphecidae" (Hymenoptera Sphecirdae \& Crabronidae) from Sicily (Italy) and Malta. Linzer biologische Beiträge, 35: 747-762.

Schmid-Egger C., Achterberg C., Neumeyer R., Morinière J. \& Schmidt S., 2017. Revision of the West Palaearctic Polistes Latreille, with the descriptions of two species - an integrative approach using morphology and DNA barcodes (Hymenoptera, Vespidae). Zookeys, 713: 53-112. https://doi.org/ 10.3897/zookeys.713.11335

Scupola A., 2019. The Strumigenys membranifera (Emery, 1860) in Salento (Apulia) and updating its occurrence in Italy (Hymenoptera Formicidae). Bollettino del Museo civico di Storia naturale di Verona, Botanica Zoologia, 43: 25-27.

Seifert B., 2016. Inconvenient hyperdiversity - the traditional concept of "Pheidole pallidula" includes four cryptic species (Hymenoptera: Formicidae). Soil organisms, 88: 1-17.

Strumia F., 2005. Insecta Hymenoptera Chrysididae. In: Ruffo S. \& Stoch F. (Eds.), Checklist e distribuzione della Fauna italiana. Memorie del Museo civico di Storia naturale di Verona (Scienze della Vita), (2) 16: 269-270.

Tenore G.C., Ritieni A., Campiglia P. \& Novellino E., 2012. Nutraceutical potential of monofloras honey produced by the Sicilian black honeybees (Apis mellifera ssp. sicula). Food \& chemical Toxicology, 50: 1955-1961. https://doi.org/10.1016/j.fct.2012.03. 067
Tomarchio S. \& Turrisi G.F., 2006. New or little known Sphecidae (Hymenoptera Aculeata) from Sicily (Italy). Linzer biologische Beiträge, 38: 953-960.

Turrisi G.F., 1999. Contributo alla conoscenza dei $\mathrm{Mu}-$ tillidae di Sicilia ed isole circumsiciliane (Hymenoptera Scolioidea). Bollettino dell'Accademia gioenia di Scienze naturali, 31 (354) [1998]: 119 155 .

Turrisi G.F., 2000. Gli Aulacidae di Sicilia, con descrizione di Pristaulacus lindae n. sp. (Hymenoptera Evanioidea). Bollettino della Società entomologica italiana, 132: 259-268.

Turrisi G.F., 2007. Revision of the Palaearctic species of Pristaulacus Kieffer, 1900 (Hymenoptera: Aulacidae). Zootaxa, 1433: 1-76.

Turrisi G.F., 2011. Diversity and biogeographical remarks on "Symphyta" of Sicily (Hymenoptera). Biogeographia, 30: 511-528.

Turrisi G.F. \& Altadonna G., 2017. A report on two alien invasive species of the genus Sceliphron Klug, 1801 from Sicily, with a brief faunistic update on the native species (Hymenoptera: Sphecidae). Biodiversity Journal, 8: 7537-7562.

Turrisi G.F. \& Rattu R., 2019. First record of the parasitoid wasp family Aulacidae (Hymenoptera) from Malta. Bulletin of the entomological Society of Malta, 10: 17-19.

Turrisi G.F., Matteini Palmerini M. \& Brothers D.J., 2015. Systematic revision and phylogeny of the genera Blakeius Ashmead, 1903 and Liomutilla André, 1907 with description of two new genera (Hymenoptera: Mutillidae, Myrmillinae). Zootaxa, 4010: 1-78. http://dx.doi.org/10.11646/zootaxa.4010.1.1

Utzeri V.J., Ribani A. \& Fontanesi L., 2018. Authentication of honey based on a DNA method to differentiate Apis mellifera subspecies: application to Sicilian honey bee (A. m. siciliana) and Iberian honey bee (A. m. iberiensis) honeys. Food Control, 91: 294-301.

Vicidomini S., 2003. Distribuzione della tribù Xylocopini (Hymenoptera: Apidae: Xylocopinae) in Italia: rassegna delle segnalazioni bibliografiche italiane. Natura bresciana, Annali del Museo civico di Scienze naturali, 33: 67-79.

Vicidomini S., Pignataro C. \& Insacco G., 2005. Xylocopini (Hymenoptera: Apidae: Xylocopinae) presenti nelle collezioni entomologiche italiane: le collezioni della Sicilia. Il Naturalista campano, 6: 1-5.

Warncke K., 1981. Beitrag zur Bienenfauna des Iran. 14. Die Gattung Halictus Latr., mit Bemerkungen uber unbekannte und neue Halictus - Arten in der Westpalaarktis und Zentralasien. Bollettino del Museo civico di Storia naturale di Venezia, 32: $67-$ 166. 